\title{
Abschreckung im Konjunktiv. Macht- und Subjektivierungseffekte von Videoüberwachung auf Demonstrationen
}

\author{
Philipp Knopp · Peter Ullrich
}

Online publiziert: 28. Mai 2019

(C) Der/die Autor(en) 2019

Zusammenfassung Die polizeiliche Videoüberwachung von Demonstrationen wird vor allem für die vermutete Abschreckungswirkung auf die Teilnahmebereitschaft an Demonstrationen kritisiert. Die vorliegende empirische Untersuchung zeigt, dass ihre Wirkungen deutlich komplexer sind. Auf Basis von Gruppendiskussionen mit rechten, linken und (links-)liberalen Demonstrierenden sowie Fußballfans werden die Macht- und Subjektivierungseffekte von Videoüberwachung systematisch in einem Grounded-Theory-Design untersucht. Videoüberwachung löst bei den Betroffenen Unsicherheiten aus, führt zu Reflexionen und produktiven Machtwirkungen wie z.B. der Übernahme polizeilicher Gefahrenkategorien durch die Demonstrierenden. Die Deutung von Videoüberwachung als Abschreckung, Repression, Schutz oder Normalität hängt einerseits mit den Staats- und Polizeibildern der Befragten zusammen und andererseits stark von der Exposition im Protestgeschehen ab. Die Bewertung von Videoüberwachung variiert zudem, je nachdem ob die Einsatzsituationen als konfliktbehaftet oder friedlich wahrgenommen werden. Der Umgang mit Videoüberwachung ist geprägt von protestspektrenspezifischen Sicherheitskulturen, die Handlungssicherheit und damit politische Handlungsfähigkeit auf Dauer herstellen sollen.

Schlüsselwörter Videoüberwachung · Abschreckung · Protest · Soziale Bewegungen · Subjektivierung · Polizei · Protest Policing · Surveillance Studies · Deutungsmuster

P. Knopp $(\square)$

Institut für Soziologie, Universität Wien, Rooseveltplatz 2, 1090 Wien, Österreich

E-Mail: philipp.knopp@univie.ac.at

P. Ullrich

Zentrum Technik und Gesellschaft, Hardenbergstr. 16-18, 10623 Berlin, Deutschland

E-Mail: ullrich@ztg.tu-berlin.de 


\title{
Subjunctive deterrence. Power and subjectification effects of video surveillance on demonstrations
}

\begin{abstract}
Public criticism of police video surveillance of demonstrations focuses on possible deterrent effects on participation in demonstrations. Empirical findings of this study, however, suggest outcomes that are more complex. Based on group discussions with left, liberal and right-wing activists as well as football fans conducted in Germany, the paper explores power and subjectification effects of video surveillance on protesters within a grounded theory research design. Video surveillance triggers uncertainty in those affected, leads to reflection and to productive power effects, one of them being the internalization of the police's threat categories by the protesters themselves. Video surveillance is interpreted as deterrence, repression, protection or normality, depending on the one hand on the interviewees' images of the state and police and on the other hand on the exposure to such measures. The evaluation of surveillance situations also depends on the protesters' assessments of them as either peaceful or not. The different ways of dealing with video surveillance are influenced by movement sector-specific security cultures, which aim to establish security of action and thus political agency.
\end{abstract}

Keywords Video surveillance $\cdot$ Deterrence $\cdot$ Protest $\cdot$ Social movements Subjectification $\cdot$ Police $\cdot$ Protest policing $\cdot$ Surveillance studies $\cdot$ Interpretation patterns

\section{Dissuasion au conditionnel. Effets de pouvoir et de subjectivation de la vidéosurveillance dans les manifestations}

Résumé L'usage policier de la vidéosurveillance dans les manifestations est surtout critiqué pour son effet dissuasif supposé sur la disposition à manifester. La présente étude empirique montre que ses effets sont nettement plus complexes. À partir de discussions de groupe avec des manifestants de droite, de gauche et libéraux (de gauche) ainsi que des fans de football, les effets de pouvoir et de subjectivation de la vidéosurveillance sont étudiés systématiquement dans une démarche de théorisation ancrée. La vidéosurveillance provoque des incertitudes chez les personnes concernées, entraîne des réflexions et des effets de pouvoir productifs comme par ex. l'adoption des catégories policières de dangerosité par les manifestants. L'interprétation de la vidéosurveillance comme dissuasive, répressive, protectrice ou normale dépend, d'une part, de la conception que les personnes interrogées ont de l'État et de la police, d'autre part et dans une large mesure, de leur niveau d'exposition dans les actions de contestation. L'appréciation de la vidéosurveillance varie en outre selon que les situations dans lesquelles la police intervient sont perçues comme conflictuelles ou pacifiques. Le rapport à la vidéosurveillance est marqué par des cultures sécuritaires spécifiques aux différents groupes contestataires destinées à garantir dans la durée un cadre sûr pour l'action et donc la capacité d'action politique. 
Mots-clés Vidéosurveillance · Dissuasion · Contestation · Mouvements sociaux · Subjectivation $\cdot$ Police $\cdot$ Gestion policière des manifestations $\cdot$ Surveillance studies · Schéma interprétatif

\section{Einleitung}

Die Videoüberwachung öffentlicher Räume ist immer wieder Thema von Debatten über Freiheit und Sicherheit. Die Kamera ist für die einen verbunden mit der Hoffnung auf eine effektive Kontrolle von Devianz, für die anderen ist sie das Symbol für eine demokratiegefährdende Überwachung schlechthin. Auch im polizeilichen Umgang mit Protest, dem Protest Policing (della Porta und Reiter 1998, S. 1), spielen Videokameras eine wichtige Rolle und sind Gegenstand ebenso heftiger Kontroversen (Ullrich und Wollinger 2011). Sie sollen der Polizei helfen, Demonstrationen besser zu managen, Straftaten zu verhindern und Verdächtiger habhaft zu werden. Überwachungskritiker/innen betonen dagegen die Gefahren für die Versammlungsfreiheit. Im Zentrum dieser kritischen Perspektiven steht ein Argument aus dem „Volkszählungsurteil“ des Bundesverfassungsgerichts von 1983 (BVerfGE $65,1)$, in dem die Richter argumentierten: ,Wer unsicher ist, ob abweichende Verhaltensweisen jederzeit notiert und [...] gespeichert, verwendet oder weitergegeben werden, wird versuchen, nicht durch solche Verhaltensweisen aufzufallen". In dieser von den Richter/innen unterstellten Wirkung staatlicher Überwachung sah das Gericht eine Begrenzung individueller Entfaltungschancen und damit eine Beeinträchtigung des demokratischen Gemeinwesens. Die Richter schufen mit dem Urteil nicht nur das Grundrecht auf informationelle Selbstbestimmung, sondern zugleich eine Argumentation, der fortan in der Rechtsprechung zur Videoüberwachung von Demonstrationen eine große Bedeutung zukommen sollte. Die Abschreckungswirkung von Videoüberwachung gefährde die durch Art. 8 des Grundgesetzes geschützte Versammlungsfreiheit (Kutscha 2011). Entsprechend gelten in Deutschland hohe Eingriffsschwellen für polizeiliche Videoüberwachung, die allerdings in der Praxis nicht immer beachtet werden. ${ }^{1}$

Der wirkmächtigen richterlichen Argumentation liegt eine quasi-soziologische These zugrunde: Überwachung löst Kontrolldruck aus, der zu Konformität und zur Abschreckung von Dissens und Dissidenz führt. Diese Annahme befindet sich weitgehend im Einklang mit der lange die Forschung zur Videoüberwachung dominierenden panoptischen Disziplinierungsthese (Foucault 1994). Doch schon die Forschung zur stationären Videoüberwachung im öffentlichen und halböffentlichen Raum hat gezeigt, dass die Effekte von Videoüberwachung weitaus komplexer sind. Für die besondere Situation des Videografierens bei Protestveranstaltungen steht eine Prüfung der Abschreckungsthese bislang jedoch aus. Daher soll in dem vorliegenden Beitrag auf Basis von Gruppendiskussionen mit von Videoüberwachung betroffenen Aktivist/innen unterschiedlicher politischer Lager systematisch der Vermutung nach-

\footnotetext{
1 Dies belegt eine Vielzahl von Gerichtsurteilen gegen die Polizei wegen unrechtmäßig eingesetzter Videoüberwachung und die kritische juristische Diskussion darum (Koranyi und Singelnstein 2011; Kutscha 2011; Arzt und Ullrich 2016).
} 
gegangen werden, dass die Abschreckungshypothese auch im Fall der Demonstrationsüberwachung nicht die Komplexität der Reaktionen und Deutungen Betroffener erfasst. Stattdessen sind aus poststrukturalistischer Perspektive heterogene Machtund Subjektivierungseffekte zu vermuten, die die Art und Weise prägen, wie sich Demonstrierende in Bezug auf die Polizei verhalten, wie sich soziale Beziehungen im untersuchten Feld gestalten und wie die Betroffenen Proteste erfahren.

Dazu werden wir zunächst unsere theoretische Perspektive darlegen und den Forschungsstand zusammenfassen (Abschnitt 2), darunter insbesondere die vorliegenden Erkenntnisse über Reaktionsweisen auf Videoüberwachung (2.1). Da bisher kaum empirische Untersuchungen zur Videoüberwachung auf Demonstrationen in Deutschland vorliegen, beziehen wir uns dabei weitgehend auf Forschungen zur stationären Videoüberwachung (,closed circuit television“, kurz CCTV). Auf dieser Grundlage plädieren wir im darauffolgenden Abschnitt (2.2) für eine Erweiterung der gouvernementalitätstheoretischen Theorien zur Videoüberwachung, um auch die vielfältigen Reaktionsweisen der von Überwachung Betroffenen integrieren zu können. Dabei wird auf den Begriff der Subjektivierungsweise zurückgegriffen, wie ihn Andrea Bührmann (2004, 2012) systematisch eingeführt hat. Diese Erweiterung ermöglicht eine enge theoretische Verbindung zwischen der Subjektivierungsform und der Subjektivierungsweise. Für unseren Gegenstand bedeutet das, empirisch zu untersuchen, wie die Protestierenden ihr Denken, Fühlen und Handeln an der Videoüberwachung und den darin zum Ausdruck kommenden Positionszuweisungen und Handlungserwartungen ausrichten. Anschließend wird der Gegenstand ,Videoüberwachung von Demonstrationen“ näher bestimmt und im Kontext gegenwärtiger Rationalitäten des selektiven Protest Policing verortet (2.3). Auf Abschnitt 3, welcher die Datengrundlage und Methoden vorstellt, folgen die empirischen Analysen. In Abschnitt 4 werden die Diskussionsgruppen, auf denen die Analyse aufbaut, vorgestellt. Dies umfasst ihre allgemeinen Charakteristika als politische Aktivist/innen sowie ihre für den Kontext der Untersuchung besonders relevanten Deutungen von Staat und Polizei. Diese haben, wie empirisch nachgezeichnet wird, Einfluss auf die Deutungen von und die praktischen Reaktionsweisen auf Videoüberwachung (Abschnitt 5 und 6). Um die verschiedenen Umgangsweisen mit polizeilichem Handeln und Videoüberwachung zu erfassen sowie deren kollektiven Charakter hervorzuheben, wird der Begriff der Sicherheitskulturen eingeführt.

\section{Theorie und Forschungsstand}

\subsection{Videoüberwachung und die Grenzen des Panoptismus}

Die Forschung zur Videoüberwachung wurde lange von Michel Foucaults (1994) Metapher des Panopticons dominiert (Haggerty und Ericson 2000; Norris 2005), einer Sozialtechnologie, die durch asymmetrische Sichtbarkeitsbeziehungen Disziplinierungseffekte auslösen und zur Internalisierung der Normen der Überwachenden durch die Überwachten führen sollte. Ullrich und Wollinger (2011, S. 27) argumentieren, dass polizeiliche Videoüberwachung auf Demonstrationen möglicherweise noch stärker als stationäre Kameras disziplinierend wirken könne, da die gleich- 
zeitige Anwesenheit von Polizist/innen das Risiko einer Bestrafung devianten Verhaltens deutlicher vergegenwärtige. Gegenüber panoptischen und auch gegenüber einigen der darauf aufbauenden gouvernementalitätstheoretischen Analysen wurden zwei zentrale Kritiken formuliert, die auch für den hier behandelten Gegenstand Berücksichtigung finden müssen.

Erstens ist zu fragen, was die in dieser Tradition analysierten Machttechnologien bei ihren Adressat/innen bewirken. Empirische Studien zeigen, dass die auf der Programmebene der Subjektformierung beispielsweise in den Skizzen des Panopticons oder in Positionspapieren für die Videoüberwachung öffentlicher Räume angenommenen Wirkungen nicht notwendig mit den beobachtbaren Effekten ineinander fallen. ${ }^{2}$ So greifen die Überwachten u.a. auf eine Reihe von „Neutralisierungstechniken“ (Marx 2003) zurück, die von demonstrativem Gehorsam über die Zerstörung der Überwachungstechnik bis hin zu aktiver „Gegenüberwachung“ (Monahan 2006) reichen. Norris und Armstrong (1999) beobachteten, dass Jugendliche die Überwachenden hinter den Kameras spielend provozierten. Autofahrer/ innen griffen Radarfallen an, weil sie sich zu Unrecht kriminalisiert fühlten (Wells und Wills 2009). Auch die Wirkungen auf Straftäter/innen und unerwünschte Personen sind vielfältig. Entgegen der populären Präventionsthese zeigten etwa Short und Ditton (1998), dass Videoüberwachung ihre Zielpersonen kaum von deviantem Verhalten abschreckte, sondern Verhaltensanpassungen und Verdrängungseffekte zur Folge hatte. Klauser (2006) wies für die Videoüberwachung eines Schweizer Rotlichtbezirks starke Gewöhnungseffekte mit Wirkungsabschwächung im Zeitverlauf nach. In einer Metaevaluation von 22 CCTV-Studien zeigten Welsh und Farrington (2002), dass Videoüberwachung nur bei der Hälfte der von ihnen ausgewerteten Studien einen signifikanten und zudem i.d. R. geringen Einfluss auf die Kriminalitätsraten hatte und ihre Wirkung in unterschiedlichen sozialräumlichen Kontexten große Unterschiede aufwies. Ähnliche Kontexteffekte sind auch bei der Videoüberwachung von Demonstrationen anzunehmen.

Zweitens stellt sich die Frage, inwiefern heutige Regierungsrationalitäten noch auf die Internalisierung von festgeschriebenen Normen zielen. Schon Foucault (1994, S. $330 \mathrm{ff}$.) und der an ihn anschließende Theoriediskurs thematisierten unintendierte Effekte des Disziplinarregimes, Momente seines Scheiterns und die Etablierung neuer dominanter Macht- und Regierungstechniken (vgl. z. B. Deleuze 1993). Die neueren Sicherheitsdispositive haben nicht mehr vorrangig die Internalisierung von Normen zum Ziel, sondern die Regulierung sozialer Prozesse durch Aktivierung und den Appell zur flexiblen Anpassung der Individuen an wechselnde Kontextanforderungen (Lessenich 2008; Heim 2013, S. 306). Die gouvernementalen Machttechniken sind daher vor allem auf die Kategorisierung von Individuen in Normalitätsklassen sowie auf ihre selbstständige Anpassung an Normalitätserwartungen ausgerichtet (Link 2005). Videoüberwachung steht idealtypisch für diese neuen Machtechniken. Krasmann (2005, S. 310) versteht sie entsprechend als „Chiffre einer neuen Gouvernementalität der Gegenwart“, da durch sie „strategische Räume“ entstehen, die Verhalten nahelegen und begrenzen, ohne eine Internalisierung festgeschriebener

\footnotetext{
${ }^{2}$ Vgl. zur entsprechenden Kritik und Erweiterung der Dispositivanalyse und der Gouvernementalitätsstu-
} dien Bührmann (2012) und Heim (2013, S. 377 ff.). 
Normen notwendig vorauszusetzen. Solcherart Überwachung appelliere vielmehr an die Interessen der Überwachten. Videoüberwachung zielt, so verstanden, nicht mehr vorrangig auf Erziehung wie die Disziplinartechniken, sondern etabliert soziotechnische „Regime der Selbstkontrolle“ (ebd., S. 318), die eine reflexive Auseinandersetzung mit dem eigenen Denken und Handeln verlangen. Diese Entwicklungen lassen sich auch im Feld des Protest Policing nachzeichnen (siehe Abschnitt 4).

Empirische Machtanalysen im Bewusstsein dieser beiden Kritiken müssen sich also hinsichtlich der erwartbaren Effekte von Videoüberwachung offen für Kontingenz und Komplexität zeigen und die Analyse auf die Reflexionen und Reaktionsweisen der Adressat/innen der neuen Machttechniken ausweiten.

\subsection{Subjektivierungsweisen von Demonstrierenden als Forschungsperspektive}

Die wenigen explorativen Studien zu den Reaktionsweisen von Demonstrierenden auf die polizeiliche Videoüberwachung von Protest offenbaren eine ähnliche Vielfalt der Umgangsweisen Betroffener wie im Fall der stationären Videoüberwachung. Betroffene äußerten das Gefühl, sich provoziert und zu Unrecht kriminalisiert zu fühlen, berichten von Ohnmacht, aber auch Wut, Aggressionen und gesteigerter Konfliktbereitschaft sowie von Ängsten bezüglich der Datenverwendung (Ullrich 2011; Shaw 2013). In verschiedenen Protestspektren existiert eine Vielzahl von auf Videoüberwachung reagierenden Gegenüberwachungspraktiken. ${ }^{3}$ Sie reichen von dezidierten politischen Kampagnen über situative und taktische Anpassungen an die Überwachung durch gleichartige Kleidung oder Vermummung bis hin zur Überwachung der Überwacher/innen mit eigener Technik. ${ }^{4}$ Zugleich verbinden selbst Überwachungskritiker/innen mit dem Objektivitätsversprechen der Videobilder die Hoffnung auf einen Schutz vor falschen Beschuldigungen (vgl. Knopp und Ullrich 2016; Ullrich und Knopp 2018). Mit ihren aufeinander bezogenen taktischen Innovationen tragen zudem beide Seiten zu einer Vertiefung der Überwachungsgesellschaft bei, die entsprechend weniger panoptisch-zentralistisch, sondern vielmehr als heterogen und auf vielfältige, disparate Bedürfnisse zurückgehend, d.h. als ,,surveillant assemblage“ gedacht werden muss (Haggerty und Ericson 2000). Allzu lineare Reiz-ReaktionsSchemata wie die Abschreckungsthese werden der Komplexität des Phänomens also nicht gerecht. Wenn Videoüberwachung hingegen als soziale Machtbeziehung verstanden wird, in der Polizist/innen auf Demonstrierende und Dritte handelnd einwirken, ${ }^{5}$ stellt sich auch die empirische Frage nach den auf die Videoüberwachung bezogenen Praktiken und Deutungen der Überwachten.

\footnotetext{
3 Der Begriff „Gegenüberwachung“ umfasst alle Praktiken, die auf die polizeiliche Anwendung von Überwachungsmaßnahmen mit dem Ziel reagieren, Ausmaß und Effekte der Überwachung zu reduzieren. Zu den videoüberwachungsbezogenen Gegenüberwachungspraktiken im engeren Sinne, die sich im Grad der Konfrontativität gegenüber der Polizei und in ihrer Ausrichtung (entweder auf das überwachende Gegenüber oder auf die eigene Gruppe) unterscheiden, vgl. Ullrich und Knopp (2018).

4 Diese als ,sousveillance“ (,Unterwachung“) bezeichnete Gegenüberwachungsmethode hat auch in den „surveillance studies“ umfangreiche Diskussionen ausgelöst (vgl. z. B. Mann et al. 2003; Neumayer und Gitte 2014; Wilson und Serisier 2010).

5 Zu diesem Begriff der Machtbeziehungen vgl. Foucault (2005, S. 251 ff.).
} 
Die dem poststrukturalistischen Machtkonzept inhärente Annahme einer Handlungsfähigkeit der Subjekte, über die Macht ausgeübt wird, kann empirisch mit dem Begriff der Subjektivierungsweisen erfasst werden. Unter Subjektivierungsweisen versteht Bührmann (2012) die Art, wie Menschen in historisch-spezifischen MachtWissens-Komplexen sich selbst und andere wahrnehmen, deuten und handeln. Die Subjektivierungsweisen konstituieren sich stets in Relation zu Subjektformierungen. Der Begriff Subjektformierung beschreibt die machtdurchdrungenen Praktiken und Diskurse der Fremdführung, mittels derer Menschen bestimmte Formen der Selbstwahrnehmung, -deutung und des Handelns lernen sollen (ebd., S. 146). Eine Forschungsperspektive, die beide Seiten der Subjektivierung berücksichtigt, nimmt die möglichen Effekte von Videoüberwachung als Machttechnik auf die Selbstund Weltverhältnisse und auf das Handeln der Überwachten nicht vorweg, sondern macht sie zu einem genuinen Gegenstand der empirischen Untersuchung. Es wäre also zu fragen, wie die Demonstrierenden ihre Protestpraktiken an der Machttechnik Videoüberwachung ausrichten (vgl. ebd., S. 159).

Die Subjektivierungs- und Machtwirkungen von Videoüberwachung sind dabei eingebettet in diskursive und nicht-diskursive Praxisgefüge der Kontrolle von Protest. In sozialen Bewegungen verändern sich Diskurse und Protestrepertoires in der Auseinandersetzung mit Techniken der Subjektformierung (Death 2010; Baumgarten und Ullrich 2016) und insbesondere mit den polizeilichen Kontrolltechniken (della Porta und Tarrow 2012). Für das eminent politische Feld der Demonstrationen ist zudem anzunehmen, dass die Verankerung der von Videoüberwachung betroffenen Aktivist/innen in politischen Strömungen für Differenzen in den Rezeptionsweisen sorgt. Zwischen Bewegungsdiskursen und -praxis vermitteln vor allem auch Deutungsmuster, die Handeln und Erfahren strukturieren (Bührmann 2012, S. 154). Deutungsmuster sind für bestimmte soziale Gruppen geltende, mit Handlungsanleitungen verbundene Interpretationsschemata der sozialen Welt, Anderer und ihrer selbst (Höffling et al. 2002). Aus dieser Perspektive stellt Videoüberwachung auch ein Handlungsproblem dar, für welches die in den Protestgruppen gültigen und verbreiteten Deutungsmuster situationsbezogene Erklärungen anbieten, u. a. über die Motive der Überwachenden, die Wirkungen auf andere Beteiligte, über angemessene Emotionen und mögliche Reaktionsweisen. Damit sind Deutungsmuster eine zentrale Komponente für die Untersuchung der kollektiven Reaktionen der Überwachten auf Videoüberwachung. Die Deutungsmuster sind nicht für alle Protestierenden identisch, sondern Bestandteil von protestspektrenspezifischen Sicherheitskulturen (Starr et al. 2008; Ullrich und Knopp 2018; vgl. dazu ausführlich Abschnitt 6). Diese sind zu verstehen als sinnhaft auf die Absicherung politischen Engagements und die Herstellung von Handlungssicherheit in der Protestsituation bezogene Ensembles aus kollektiven Wissensformen und normativ aufgeladenen Praktiken, die sich in den vielschichtigen Konstellationen zwischen Demonstrierenden, der Polizei und ihren Publika etabliert haben und verändern.

Der Beitrag hat also in Ergänzung zur klassischen gouvernementalitätstheoretischen Analyse von Videoüberwachung als Regierungs- und Machttechnik einen doppelten empirischen Fokus: auf die Deutungsmuster von Videoüberwachung einerseits und die Kulturen des Umgangs mit Videoüberwachung andererseits. 


\subsection{Subjektformierung: Videoüberwachung und selektives Protest Policing}

Die Videoüberwachung von Demonstrationen erfolgt i.d.R. durch mobile Kameras von Videoteams oder aus Einsatzfahrzeugen. Sie dient im Fall von Übersichtsaufnahmen aus der Distanz (aus Hubschraubern, speziellen Fahrzeugen oder Drohnen) primär der Übersicht über das Geschehen vor Ort und, im deutlich häufigeren Einsatzszenario, insbesondere der Beweissicherung. Dieser Einsatz lässt sich als soziotechnischer Prozess der Hervorbringung von audiovisueller Evidenz verstehen. In diesem werden schrittweise und aufeinander aufbauend (von der Bereithaltung der Kameras bei Versammlungen, über die Aufnahmeentscheidung, Fokuswahl, Bildbearbeitung und Interpretation bis zur Verwendung oder Löschung) Handlungsresultate objektiviert, was zugleich die Kontingenz ihres Zustandekommens verschleiert und so eine „Definitionsmachtkette“ konstituiert (Ullrich 2018). Die Definitionsmacht der Polizei (Feest und Blankenburg 1972) beruht auf dem juristischen und soziologischen Ermessensspielraum und damit nicht zuletzt auch auf vor- und auBerrechtlichen polizeilichen Normvorstellungen, stereotypen Klassifikationen von Demonstrierenden und teils politisch-bewertenden Verdachtskategorien, die insbesondere Auswirkungen auf die Wahrscheinlichkeit haben, videoüberwacht zu werden (Arzt und Ullrich 2016; Ullrich 2018).

Die Videoüberwachungspraxis der Polizei ist eingebettet in den historisch spezifischen Macht-Wissens-Komplex selektiver und situativ-präventiver Kontrollpraktiken, die Demonstrierende in Gefährder/innenklassen unterscheiden und auf dieser Klassifizierung aufbauend unterschiedlich behandeln. Diese als „selektives Policing“ bezeichnete Grundausrichtung löste in Deutschland und Europa seit den 1970- und 1980er-Jahren die bis dahin dominierende „eskalative Stärke“ (streng legalistisches Vorgehen mit niedrigschwelligem und unterschiedslosem Gewalteinsatz) als dominante Strategie des Protest Policing ab (vgl. della Porta et al. 2006).

Maßgeblich für die Wende hin zum selektiven Protest Policing waren sowohl die Rechtsprechung des Bundesverfassungsgerichts als auch wissenschaftlich begründete Einsatzkonzeptionen, die auf der Notwendigkeit der Unterscheidung zwischen „guten“ und „bösen“ Demonstrierenden beruhen. Zentral für die polizeilichen normativen Erwartungen an das Verhalten der Demonstrierenden und damit für die Subjektivierungsform des Demonstrierenden sind Friedlichkeit, Kooperation mit der Polizei und die deutliche Distanzierung von militanten Protestierenden. Verschiedentlich wiesen empirische Untersuchungen auch darauf hin, dass politische Distanz von Polizist/innen zu bestimmten Versammlungen die Wahl härterer Policing-Strategien veranlassen können (della Porta und Reiter 1998; della Porta et al. 2006; Ullrich 2017).

Wichtigster juristischer Referenzpunkt für das selektive Protest Policing ist in Deutschland das Differenzierungsgebot aus dem Brokdorf-Beschluss des Bundesverfassungsgerichts von 1985 (BVerfGE 69, 315), welches der Polizei grundsätzlich eine Demonstrationsfreundlichkeit abverlangt, selbst wenn Teile der Demonstrierenden militant agieren. Eine wissenschaftliche Grundlage für ein solches Polizeihandeln findet sich in gegenwärtig einflussreichen Policing-Konzeptionen wie dem „elaborated social identity model of crowd behaviour" (Rikspolisstyrelsen 2013, S. 8; Schreiber und Adang 2008). Diese Modelle empfehlen die Isolation von „Stö- 
rern“ und zielgerichtete Interventionen gegen diese, um Frontstellungen zwischen größeren Menschenmengen und der Polizei zu vermeiden; zudem sollen friedliche Demonstrierende und deren Anliegen unterstützt und für ,Self-Policing "6 aktiviert werden (Reicher et al. 2007; grundlegend Feeley und Simon 1992). ${ }^{7}$

Vor Demonstrationen sammelt die Polizei Informationen über zu erwartende Protestspektren, um ihre Einsatztaktik und -stärke darauf abzustimmen. Dazu werden zunehmend auch technische Überwachungssysteme eingesetzt (vgl. della Porta et al. 2006; Gillham 2011). Im Zuge des Bedeutungszuwachses der Klassifizierung von Demonstrierenden formalisierten die deutschen Polizeien auch die entsprechenden Kategoriensysteme. Im Einsatz selbst basiert die Kategorisierung von Demonstrierenden vorrangig auf äußeren Merkmalen (insbesondere Kleidung). Die Demonstrierenden werden Gefahrenklassen zugewiesen, die oft einem dreistufigen Ampelsystem entstammen (gewalttätig, unter Umständen gewalttätig, friedlich; Dießelmann 2015, S. 10). Die Kategorisierung dient zugleich der praktischen Handhabung und der statistischen Erfassung von Protestgruppen. Um den selektiven Umgang zu realisieren, kam es auch zu organisationsstrukturellen Innovationen, u. a. den Beweissicherungs- und Festnahmeeinheiten (BFE), die gezielt und räumlich eng begrenzt intervenieren und Festnahmen durch Videoaufnahmen zugleich beweissichernd durchführen. Insbesondere beim Policing von Großprotesten werden aber oft weiträumige Einschließungsmaßnahmen, sogenannte Kessel, angewandt, um mitunter hunderte als gefährlich eingeschätzte Demonstrierende vom Rest der Versammlung zu separieren (vgl. u. a. Bernhardt 2014).

Videoüberwachung fügt sich in die strategischen Machttechniken des selektiven Protest Policing als historisch spezifische Subjektformierung ein, weil sie (im Fall von Übersichtsaufnahmen) das Demonstrationsmanagement erleichtert, indem polizeilich relevante Gruppen identifiziert und Einsatztaktiken darauf angepasst werden können, und weil sie (durch Beweissicherungsaufnahmen) zeitlich verzögerte Verhaftungen in weniger konflikthaften Situationen (z. B. nach dem Ende statt während einer Demonstration) ermöglicht. Die unmittelbare Abschreckung von unerwünschtem Verhalten, die als Topos die kritische Debatte bestimmt, ist somit zwar ein polizeiliches Motiv für den Kameraeinsatz, aber nur eines unter vielen. Neben den bereits genannten bilden die Dokumentation des eigenen Vorgehens, die Imagepflege und die Einsatzauswertung weitere Motive (Ullrich 2018, S. 331 f.).

\section{Datengrundlage und Methode}

Die Datenbasis unserer empirischen Untersuchung bilden zwölf 60- bis 90-minütige problemzentrierte Gruppendiskussionen mit von polizeilicher Videoüberwachung

\footnotetext{
${ }^{6}$ Dies umfasst in Einzelfällen auch die Unterstützung von Veranstaltungen, die der Polizei als friedliche Gegenveranstaltungen gegen vermeintlich gewalttätige andere Versammlungen gelten, aber auch den Einsatz von Ordner/innen bei der Versammlung. Prinzipiell sollen „,friedliche“ Demonstrierende ermuntert werden, sich von ,gewaltbereiten “ Demonstrierenden zu distanzieren und im Fall von Auseinandersetzungen die Strafverfolgung zu unterstützen.

7 Das bedeutet freilich kein Verschwinden der Anwendung von Zwangsmaßnahmen und körperlicher Gewalt, insbesondere gegen Demonstrierende, die der Anrufung zur Selbstkontrolle nicht nachkommen.
} 
betroffenen Aktivist/innen, die überwiegend in den Jahren 2015/16 geführt wurden. Die Teilnehmer/innen gehören linken, rechten und links-liberalen Protestspektren sowie in einem Fall der Fußball-Ultraszene an. ${ }^{8}$ Letztere wurden in die Untersuchung aufgenommen, da Sportveranstaltungen teilweise ähnliche Überwachungsund Policing-Situationen aufweisen wie politische Versammlungen, ${ }^{9}$ aber politische Deutungsmuster für sie weniger konstitutiv sind. Die Ultra-Gruppe ermöglicht also instruktive Vergleiche, die den möglichen Einfluss politischer Deutungsmuster erhellen können. Der Feldzugang erwies sich sowohl bei den linken Gruppen und der liberalen Gruppe als auch bei den Ultra-Fans als unkompliziert. Dazu trug auch die Zusicherung von umfangreichen Datenschutzmaßnahmen bei. Bei den rechten Gruppen gestaltete sich der Zugang deutlich schwieriger. Trotz wiederholter Anfragen konnten militante Rechte oder Mitglieder neonazistischer Organisationen nicht für die Gruppendiskussionen gewonnen werden.

Aus den insgesamt zwölf Gruppen wurden in einem theoretischen Sampling sechs Kontrastfälle für die Feinanalyse ausgewählt, die charakteristisch für das jeweilige Spektrum sind (Strauss und Corbin 1998, S. $201 \mathrm{ff}$.). Um eine möglichst umfassende Darstellung zu gewährleisten, wurden alle politischen Grundströmungen in das Sample aufgenommen. Nach einer ersten Materialsichtung wurden die Gruppen ausgewählt, in denen sich divergierende Deutungen und Handlungsweisen abzeichneten (vgl. die Gruppenübersicht in Tab. 1); die Einbeziehung weiterer Gruppen hätte nicht zur Erhöhung der konzeptuellen Repräsentativität (ebd., S. 214) beigetragen. Es handelt sich um überwiegend „,natürliche Gruppen“ mit gemeinsamer beziehungsweise sich überschneidender politischer Praxis; dies baut Artikulationshemmnisse (beispielsweise bei Berichten über illegale Handlungen, aber auch im Falle von Dissens in der Gruppe) ab und stärkt die interne Deutungshomogenität.

Die Gruppendiskussion als Erhebungsverfahren ermöglicht die Rekonstruktion von Alltagsdiskursen. Dadurch können die Sinngebungen, kollektiven Normen und Praktiken der Befragten erhoben werden. Weil die Teilnehmenden spontan aufeinander reagieren, treten auch nichtöffentliche Aspekte und Theorien über ein Thema zutage (vgl. Kitzinger 1994, S. 113). Insbesondere für die Untersuchung der auf Videoüberwachung bezogenen Deutungsmuster und Subjektivierungseffekte ist zudem von Vorteil, dass in Gruppendiskussionen sowohl diskursiv vermitteltes Wissen, Alltagserfahrungen und Praxisreflexionen als auch deren möglicherweise konfligierenden Beziehungen zur Sprache kommen.

Die Gruppendiskussionen begannen mit einer Einstiegsfrage zum politischen Engagement der Anwesenden. Um dann zur Diskussion zu animieren, zeigten die Erheber/innen Videos mit einer an die jeweilige Gruppe angepassten Demonstrationssituation mit sichtbarer polizeilicher Videotechnik. Die Diskussionsdynamik war größtenteils selbstläufig. Die Erheber/innen beschränkten sich auf Verständnisfragen

\footnotetext{
8 Die Gruppen waren hinsichtlich der Geschlechter unterschiedlich zusammengesetzt: In der liberalen und rechten Gruppe waren nur Männer, in der Ultra-Gruppe waren mehrheitlich Männer und in den linken Gruppen waren Männer und Frauen in etwa gleich stark vertreten.

9 Auch hinsichtlich der Ausdrucksformen von Ultra-Fans gibt es Gemeinsamkeiten mit den anderen Protestgruppen wie z. B. demonstrationsähnliche sogenannte Fanmärsche mit Transparenten und Sprechchören.
} 
Tab. 1 Grundcharakteristika der Gruppen und ihre Deutungsmuster der Polizei und Staatsverständnisse

\begin{tabular}{|c|c|c|c|c|c|}
\hline Gruppe & $\begin{array}{l}\text { Politische } \\
\text { Grundströ- } \\
\text { mung }\end{array}$ & Themenfelder & Aktionsrepertoire & $\begin{array}{l}\text { Deutungsmuster } \\
\text { der Polizei }\end{array}$ & Staatsverständnis \\
\hline $\begin{array}{l}01 \\
\text { Ultra-Fuß- } \\
\text { ballfans }\end{array}$ & - & Fankultur & $\begin{array}{l}\text { Choreografien, } \\
\text { Fanmärsche, } \\
\text { kleinere Regel- } \\
\text { übertretungen } \\
\text { im Stadion }\end{array}$ & $\begin{array}{l}\text { Übermächtiger } \\
\text { Gegner }\end{array}$ & $\begin{array}{l}\text { Repressiver } \\
\text { Staat }\end{array}$ \\
\hline $\begin{array}{l}02 \\
\text { Linksradikale } \\
\text { Gruppe }\end{array}$ & links & $\begin{array}{l}\text { Antifaschismus, } \\
\text { Antiras- } \\
\text { sismus, } \\
\text { Freiraumpoli- } \\
\text { tik }\end{array}$ & $\begin{array}{l}\text { Demonstrationen, } \\
\text { Blockaden, } \\
\text { ziviler } \\
\text { Ungehorsam }\end{array}$ & $\begin{array}{l}\text { Übermächtiger } \\
\text { Gegner }\end{array}$ & $\begin{array}{l}\text { Repressiver } \\
\text { Staat }\end{array}$ \\
\hline $\begin{array}{l}03 \\
\text { Linksradikale } \\
\text { Gruppe }\end{array}$ & links & $\begin{array}{l}\text { Antifaschismus, } \\
\text { Feminismus, } \\
\text { Antikapi- } \\
\text { talismus, } \\
\text { Wohnraumpo- } \\
\text { litik }\end{array}$ & $\begin{array}{l}\text { Demonstrationen, } \\
\text { Blockaden, } \\
\text { militante } \\
\text { Aktionen }\end{array}$ & $\begin{array}{l}\text { Übermächtiger } \\
\text { Gegner }\end{array}$ & $\begin{array}{l}\text { Repressiver } \\
\text { Staat }\end{array}$ \\
\hline $\begin{array}{l}04 \\
\text { Linke } \\
\text { Jugend- } \\
\text { gruppe }\end{array}$ & links & $\begin{array}{l}\text { Antifaschismus, } \\
\text { Antiras- } \\
\text { sismus, } \\
\text { Internationa- } \\
\text { lismus }\end{array}$ & $\begin{array}{l}\text { Demonstrationen, } \\
\text { Blockaden }\end{array}$ & $\begin{array}{l}\text { Auf dem } \\
\text { rechten Au- } \\
\text { ge blinder } \\
\text { Schiedsrich- } \\
\text { ter }\end{array}$ & $\begin{array}{l}\text { Bedingt ver- } \\
\text { trauenswürdi- } \\
\text { ger Rechtsstaat }\end{array}$ \\
\hline $\begin{array}{l}05 \\
\text { Liberale } \\
\text { Gruppe }\end{array}$ & liberal & $\begin{array}{l}\text { Queerpolitisch, } \\
\text { z.T. Antifa- } \\
\text { schismus }\end{array}$ & $\begin{array}{l}\text { Demonstrationen, } \\
\text { Mahnwachen }\end{array}$ & $\begin{array}{l}\text { Freund und } \\
\text { Helfer }\end{array}$ & $\begin{array}{l}\text { Sehr vertrau- } \\
\text { enswürdiger } \\
\text { Rechtsstaat }\end{array}$ \\
\hline $\begin{array}{l}06 \\
\text { Rechte } \\
\text { Gruppe }\end{array}$ & rechts & $\begin{array}{l}\text { Gegen Islam } \\
\text { und Migration }\end{array}$ & $\begin{array}{l}\text { Demonstrationen, } \\
\text { ziviler Unge- } \\
\text { horsam }\end{array}$ & $\begin{array}{l}\text { Politisch } \\
\text { verein- } \\
\text { nahmter } \\
\text { Beschützer }\end{array}$ & $\begin{array}{l}\text { Beschädigter } \\
\text { Rechtsstaat }\end{array}$ \\
\hline
\end{tabular}

und reflektierende Nachfragen zu den im Verlauf der Diskussion angerissenen Themen. In den seltenen Fällen, in denen die Diskussionen stockten, wurden zusätzliche narrationsgenerierende Fragen gestellt oder als zweiter Stimulus ein Gesetzestext zur Videoüberwachung von Demonstrationen vorgelegt. Die Tonaufnahmen wurden transkribiert $^{10}$, anonymisiert und mit Unterstützung der CAQD-Software MAXQDA ausgewertet. Die Auswertung in einem Grounded-Theory-Design erfolgte zunächst im ,open coding“, dann selektiv und axial im Sinne der Fragestellung ausgerichtet an den auftretenden Deutungsmustern und Handlungsreflexionen.

\section{Charakterisierung der Diskussionsgruppen sowie ihrer Deutungen von Staat und Polizei}

Die Analyse der Gruppendiskussionen ergab, dass die Deutungen von Videoüberwachung bei Demonstrationen mit dem jeweiligen Verhältnis der Diskussionsteil-

10 Die im Text wiedergegebenen Zitate wurden zwecks besserer Lesbarkeit sprachlich behutsam geglättet. 
nehmer zum Staat und mit ihren allgemeinen Deutungen polizeilichen Handelns konvergieren: Nicht videoüberwachungsbezogene Deutungsmuster von Motiven und Wirkungen polizeilichen Handelns prägen auch die Interpretationen von Videoüberwachung. Um diese Einbettung der Deutungsmuster von Videoüberwachung nachvollziehbar zu machen, werden im Folgenden die sechs Diskussionsgruppen charakterisiert. Dies beinhaltet neben ihrem Selbstverständnis, ihren politischen Themenfeldern und ihrer Zugehörigkeit zu politischen Strömungen (Liberale, Linke, Rechte und Ultra-Szene) insbesondere ihr grundsätzliches Verständnis vom Staat und die eng damit verbundenen Polizeibilder (vgl. Tab. 1). Vier Deutungsmuster der Polizei und vier Staatsverständnisse traten in den Gruppendiskussionen zutage und bilden die Grundlage der folgenden Typologie der befragten Gruppen.

Die linksradikalen Gruppen (02, 03) und die Gruppe der Ultra-Fans (01) zeigten ein skeptisches bis strikt ablehnendes Verhältnis zur Polizei als Institution eines repressiven Staates. Alle deuten die Polizei als einen übermächtigen Gegner. Die politischen Themenfelder der linksradikalen Gruppen sind breit gefächert. Dazu zählen Feminismus, Antifaschismus, Mieten und Freiraumpolitik. Eine der Gruppen (03) kann dem linksradikal-autonomen Spektrum zugeordnet werden und versteht sich als antietatistisch und antikapitalistisch. Diese Gruppe geht z. T. bewusst Auseinandersetzungen mit Neonazis und der Polizei ein. Insbesondere diese Gruppe ist während der Gruppendiskussion sehr um eine rationale und reflektierte Analyse des eigenen Handelns und des Handelns Anderer bemüht. Die zweite linksradikale Gruppe (02) benennt neben Demonstrationen auch Blockaden gegen rechte Demonstrationen als probates politisches Ausdrucksmittel. Die Ultra-Fans organisieren Choreografien in Fußballstadien sowie Fanmärsche und nehmen an szenespezifischen Demonstrationen teil, auf denen sie für die Rechte von Fans eintreten. Sie bezeichnen diese Demonstrationen aber selbst als nicht politisch und betonen, keine strafrechtlich relevanten Handlungen, jedoch kleinere Regelübertretungen zu begehen. Die linksradikalen Gruppen und die Ultra-Gruppe deuten das Handeln der Polizei und ihre stets hohe Präsenz als Machtdemonstration des repressiven Staates, der unliebsame Versammlungen unterdrücken, durch Kriminalisierung politisch delegitimieren und stigmatisieren wolle - selbst, wenn sie friedlich seien. Zwar wird ein Eingreifen der Polizei bei Auseinandersetzungen durchaus als Teil eines normalen und gewohnten Konfliktverlaufs angesehen, zu dem auch Demonstrierende beitragen; die Eskalation von Konflikten führe die Polizei aber teilweise durch Provokationen selbst bewusst herbei. Versammlungen erfahren diese Gruppen als Schauplatz eines Kräftespiels mit einer überlegenen und gewaltaffinen Polizei. Alle drei Diskussionsgruppen berichten detailliert von der permanenten Präsenz von Videoüberwachung bei ihren Protesten. Polizei und Videoüberwachung sind auch Themen in ihren Alltagsgesprächen.

Ein kritisches, aber weniger grundsätzlich ablehnendes Deutungsmuster von Polizei und Staat dominiert in der linken Jugendgruppe (04), deren Hauptthemenfelder Antifaschismus, Internationalismus und Antirassismus sind. Die Polizei deuten sie zumindest im eigenen Bundesland - als auf dem rechten Auge blinder Schiedsrichter. Die Aktivist/innen teilen die Erfahrung von Provokationen, Polizeigewalt und häufig umfangreicher Polizeipräsenz mit den linksradikalen Gruppen. Allerdings wird nicht der Staat an sich als feindselig gedeutet, sondern einzelne Polizist/innen, deren rechte Einstellungen sich in Gewalt gegen Linke und im Schutz für rechte Demons- 
trierende durch unterlassene Strafverfolgung äußerten. Die politische Distanz der Polizei zur Linken erklären die jungen Aktivist/innen aber auch durch einsatzverursachte Belastungen wie Überstunden und durch Aspekte des Auftretens der eigenen Seite (geringer Respekt für die Polizei, Gewalt). Es dominiert dennoch das Gefühl, ungerecht und vor allem verglichen mit rechten Demonstrierenden schlechter behandelt zu werden. Allerdings schreiben die jungen Linken der Polizei als Institution eine im Grundsatz positive Rolle als Schiedsrichter zu, die sie jedoch im eigenen Bundesland nicht erfülle. Die Regionalisierung und Personalisierung von erfahrenen Missständen erlaubt es, die Deutung des Rechtsstaats als bedingt vertrauenswürdig aufrecht zu erhalten. Positive Erfahrungen mit der Polizei bleiben aber auch in dieser Diskussion randständig. Die Gruppe thematisiert Videoüberwachung ohne Umschweife, wobei der Fokus der Diskussion wiederholt zum Themenkomplex der vermuteten rechten Gesinnung der Polizeibeamt/innen zurückkehrt.

Bei den links-liberalen Demonstrierenden (05, im Folgenden kurz als ,liberal“ bezeichnet), die sich v. a. im queerpolitischen Themenbereich und für Homosexuellenrechte engagieren, gestaltet sich die Beziehung zur Polizei kooperativ und vertrauensvoll. Sie wird als Freund und Helfer erfahren, ohne den der eigene friedliche Aktivismus homophoben Angriffen ausgesetzt sein könnte. Deutschland schätzen sie im Vergleich zu autoritären Staaten als sehr vertrauenswürdigen Rechtsstaat. Zwar vermuten die liberalen Demonstrierenden auch innerhalb der Polizei rechte und homophobe Einstellungen, die sich gegen die eigene Gruppe richten. Im Allgemeinen nehmen die Aktivist/innen aber eine hohe Akzeptanz für ihre politischen Ziele wahr, was sich im polizeilichen Umgang mit ihren Versammlungen zeige. Ihre Sicht auf die Polizei ist also nicht vorbehaltlos unkritisch, und es wird auch in Erwägung gezogen, sich im Falle des Auftretens von ,zu eingreifenden“ Maßnahmen $\mathrm{zu}$ wehren. $\mathrm{Zu}$ betonen ist, dass diese Gruppe bisher bei eigenen Versammlungen nicht mit polizeilicher Videoüberwachung konfrontiert war. Die Diskussion entfaltet sich daher zunächst über das Thema der stationären Videoüberwachung öffentlicher Plätze, mit dem sie mehr Alltagserfahrungen verbinden. Deren Bewertung ist in der Gruppe umstritten. Einige Teilnehmer/innen heben den Sicherheitsgewinn hervor, während andere die Einschränkung von Freiheitsrechten kritisieren. Davon ausgehend erörtern sie die Situation auf Demonstrationen angesichts mangelnder eigener Erfahrungen abstrakt und spekulativ.

Die rechten Aktivist/innen aus dem völkisch-elitären Spektrum (06) deuten die Polizei ambivalent als politisch vereinnahmten Beschützer. Das Verhältnis zur Polizei und zum Staat als „Idee“ ist affirmativ, die Arbeit der Polizei schütze und ermögliche die eigenen Versammlungen, da man in der Konfrontation mit den politischen Gegner/innen unterlegen sei. Zu diesen zählten namentlich die Medien und „die Antifa“, mit denen man in einem „Zweifrontenkrieg“ stehe. Die Anerkennung für die Polizei beruht auf ihrer repressiven Stärke, die sich im Demonstrationsalltag meist gegen Linke richte. Nur in Ausnahmesituationen nehmen die rechten Demonstrierenden das Handeln der Polizei als ,unprofessionell“ oder ungerecht wahr, z. B. wenn sich Maßnahmen gegen die eigene Gruppe richten oder wenn die Polizei antifaschistische Blockaden nicht mit allen Mitteln verhindert. Ein solches Verhalten der Polizei zum eigenen Nachteil, das auch Aggressionen mit verantworte, erklären sich die rechten Protestierenden mit einer Einflussnahme der Politik. So zeichnet 
sich bei dieser Gruppe ein ambivalentes Verhältnis zum polizeilichen Handeln ab, das in konkreten Narrativen v.a. dann positiv bewertet wird, wenn die Polizist/ innen im Sinne der situativen Interessen des eigenen Protestspektrums handeln. Das Engagement der Gruppe gelte der „Wiederherstellung von Rechtsstaatlichkeit“, die „illegale Einwanderung“ und „Überfremdung“ „,nachhaltig“ negativ beeinflussten. Ihre Distanz zum Staat, der sich ihnen als beschädigter Rechtsstaat darstellt, drückt sich in der Annahme aus, dass gegenwärtige politische Eliten gegen die Interessen und Einstellungen der Gruppe agierten, die sie als Interessen der Bevölkerung und als Rechtsstaatsprinzip generalisieren. Der „Idee des Staates“ sei man aber weiterhin verpflichtet. Die rechte Gruppe verfügt nur über wenige Erfahrungen mit repressivem polizeilichen Vorgehen und Videoüberwachung. Die Rede über Videoüberwachung bleibt meist abstrakt oder beschränkt sich auf Forderungen nach mehr Überwachung der politischen Gegner/innen. Den Diskussionsverlauf dominieren stattdessen, wie auch bei einer anderen interviewten rechten Gruppe, politische Themen der Neuen Rechten, wie Migration, die Berichterstattung der Medien und das Verhältnis zu den politischen Eliten.

Die Diskussionsgruppen interpretieren polizeiliches Handeln oft als politisch motiviert. Ein versammlungsfreundliches Verhalten bringen sie eher mit Zustimmung zu den eigenen politischen Zielen in Verbindung (05), während sie ein repressives Vorgehen als Anzeichen für eine Gegnerschaft der Polizei als Institution des Staates gegen politisch linksradikale Strömungen $(02,03)$ oder Bürger/innen im Allgemeinen (01) deuten. Einige personalisieren die wahrgenommene Gegnerschaft, indem sie auf rechte Einstellungen bei der Mehrheit der Polizist/innen zurückgeführt (04) oder eine politisch verordnete Repression ,,von oben“ angenommen wird (06).

Bereits in diesen allgemeinen Aussagen über die Polizei und den Staat zeigen sich Muster, die auch für die im Folgenden dargestellten Deutungen von Videoüberwachung charakteristisch sind. Polizeiliche Eingriffe in die Versammlung und hohe Polizeipräsenz erfahren die Demonstrierenden als Missachtung ihres politischen Anliegens und Delegitimierung der eigenen politischen Position. Wenn die Teilnehmer/innen der Gruppendiskussionen in einem solchen polizeilichen Handeln keine strafrechtliche oder sicherheitsbezogene Motivation erkennen können, interpretieren sie diese Maßnahmen als politisch motivierte Kriminalisierung des eigenen politischen Spektrums. Kooperative und zurückhaltende Formen polizeilicher Kontrolle deuten sie hingegen als Zustimmung zum politischen Anliegen der so behandelten Versammlungen. Alle Gruppen neigen in diesem Sinne zu einer Politisierung polizeilichen Handelns. Zugleich deuten die Diskutant/innen die repressiven Eingriffe auch als von der Polizei betriebene Entpolitisierung ihrer Proteste vor den Augen der Öffentlichkeit. Der polizeiliche Umgang mit Protest beeinflusst also die Erwartung politischer Selbstwirksamkeit der Aktivist/innen. Das Spannungsverhältnis von wahrgenommener Kriminalisierung und Entpolitisierung des eigenen Handelns durch die Polizei und Politisierung polizeilichen Handelns durch die Diskutant/innen durchzieht auch die Deutungen von Videoüberwachung. Der durch die Polizei repräsentierte Staat wird damit selbst als politischer Akteur wahrgenommen und mithin zum expliziten Kontrahenten. Die antifaschistische Jugendgruppe weicht davon ab, indem sie die Bevorzugung rechter Versammlungen als regionale bzw. individuelle Idiosynkrasie deutet. 
Tab. 2 Deutungsmuster von Videoüberwachung im Überblick

\begin{tabular}{lll}
\hline Deutungsmuster & Subtypen & Vorkommen in den Gruppen \\
\hline $\begin{array}{l}\text { Abschreckung } \\
\text { Politische Repression }\end{array}$ & $\begin{array}{l}\text { Kriminalisierung der eigenen } \\
\text { Gruppe }\end{array}$ & $\begin{array}{l}\text { Ultra-Fans, alle linken Gruppen } \\
(01-04)\end{array}$ \\
& $\begin{array}{l}\text { a) als Anzeichen für politische } \\
\text { Vereinnahmung der Polizei }\end{array}$ & Rechte (06) \\
& b) als Anzeichen von rechter & Linke Jugendgruppe (04), z. T. \\
& Gesinnung von Polizist/innen & Linksradikale (03) \\
& Provokation & Alle \\
& Datensammlung & Alle \\
& & Linksradikale (02, 03), linke \\
& & Jugendgruppe (04) \\
Konflikt & & Liberale (05) und Rechte (06) \\
Schutz & &
\end{tabular}

\section{Unsicherheit und die Deutung von Videoüberwachung}

Schon Foucault (1994) identifizierte die Verunsicherung der Überwachten angesichts des Blicks der Überwachenden als wesentliche Wirkungsweise panoptischer Sichtbarkeitsbeziehungen. Solcherart Verunsicherungen werden auch in allen Gruppendiskussionen als Grunderfahrung der Befragten sichtbar. In der Überwachungssituation selbst ist ihnen oft nicht ersichtlich, ob die mitgeführten Polizeikameras tatsächlich filmen. Eine linksradikale Gruppe (03) diskutierte, ob die Kamera ein Anzeichen für drohende weitere polizeiliche Interventionen oder eine symbolische Einschüchterungsmaßnahme ist. Die Auswertung des Videomaterials wird tendenziell als bedrohlich empfunden und ist Gegenstand von Spekulationen. Auch wenn die diesbezüglichen begrenzten rechtlichen Befugnisse der Polizei in einigen Gruppen (01-03) durchaus bekannt sind, misstrauen sie der polizeilichen Rechtstreue und vermuten eine weit umfänglichere Datenspeicherung und die Verwendung dieser Daten für repressive Zwecke.

Die Deutungsmuster von Videoüberwachung helfen den Demonstrierenden vor dem Hintergrund dieser Unsicherheit, den Kameraeinsatz einzuordnen und wiederum Handlungssicherheit zu gewinnen. Dabei fließen Selbstbilder und die jeweiligen Beziehungen zu Staat und Polizei, aber auch zu Dritten (politische Kontrahent/innen, Publika) in die Deutungen von Videoüberwachung ein. Die vier identifizierten übergreifenden Deutungsmuster sind entweder vorrangig diskursbezogen (Abschreckung) oder vorrangig situationsgebunden (politische Repression, akzeptierte Normalität im Konflikt und Schutz). Das Deutungsmuster politische Repression beinhaltet drei Subtypen (Kriminalisierung, Provokation, Datensammlung), die es auf bestimmte Überwachungsmotive und -wirkungen hin präzisieren; diesen Ausprägungen ist gemein, dass die Überwachungspraxis als illegitim bewertet wird - im Gegensatz zum Deutungsmuster akzeptierte Normalität im Konflikt und Schutz (vgl. Tab. 2). Auffällige Übereinstimmungen über die Gruppen hinweg gibt es in der negativen Bewertung von Videoüberwachung in konfliktarmen Situationen. Deutliche Unterschiede zeigen sich aber in den auf Videoüberwachung bezogenen Forderungen. Sie reichen von kompletter Abschaffung (Linksradikale) über Forderungen nach einer egalitä- 
ren Verteilung von Überwachung (linke Jugendgruppe) bis hin zu Forderungen nach einer zielgerichteten Überwachung von Straftäter/innen (Liberale) oder politischen Gegner/innen (Rechte).

\subsection{Abschreckung}

Fast alle Gruppendiskussionen nehmen den Topos der Abschreckung auf. ${ }^{11}$ Zum einen vermuten die Demonstrierenden die Behinderung der Teilnahme an Versammlungen durch abschreckende Kameras als polizeiliches Einsatzmotiv. Videoüberwachung und polizeiliche Zwangsmaßnahmen gelten als Ausdruck eines undemokratischen Umgangs mit sich versammelnden Bürger/innen (01) oder als Ausdruck einer spezifischen Gegnerschaft $(02,03,04,06)$. Zum anderen wird das Fernbleiben von Bekannten der Interviewten sowie von demonstrationsunerfahrenen Personen mit der angenommenen abschreckenden Wirkung von Videoüberwachung erklärt (04, 06).

Die Demonstrierendengruppen bewerten die Abschreckungswirkung jedoch unterschiedlich. Während in den linken Gruppen und bei den Ultra-Fans eine negative Beurteilung dominiert, die vor allem die Einschränkung demokratischer Rechte kritisiert, vermuten die liberalen Demonstrierenden auch eine positive präventive Wirkung gegenüber potenziellen Straftäter/innen. Die rechten Diskutant/innen sehen eine ,im positiven Sinne abschreckende Wirkung“(06) im Hinblick auf Gegendemonstrierende - im Gegensatz zur als repressiv empfundenen Abschreckungswirkung auf die eigene Gruppe.

Bei den dargestellten Deutungen handelt es sich um Allgemeinurteile und Einschätzungen des Verhaltens Dritter. In Erzählungen über die eigenen Erfahrungen und Erlebnisse hat dies keine Entsprechung: Abschreckungswirkungen werden stark eingeschränkt, explizit verneint oder nur in einem hypothetischen Erzählmodus erörtert. So überlegt ein rechter Demonstrierender: „Wenn ich [...] vor der Entscheidung stände, soll ich das tun [eine Rede halten; d. A.] oder nicht und ich weiß, da ist jetzt eine Polizeikamera auf mich gerichtet, finde ich das auch einschüchternd“ (06). In dieser Beschreibung ruft er das diskursmächtige Deutungsmuster Abschreckung auf und präsentiert es als womöglich handlungsleitend, ohne dass dem eigene Erfahrungen zugrunde liegen.

Von einer tatsächlich abschreckenden Wirkung auf die Befragten selbst wird deutlich seltener berichtet. Eine linksradikale Gruppe (03) spricht von besonders vorsichtigem Agieren, das deutlich fokussierte Kameras auslösten. Sie konstatiert auch Verunsicherung nach Handlungen, die augenscheinlich gefilmt wurden. Zudem diagnostizieren sie eine „krasse Kameraparanoidität“ (03) in ihrem Protestspektrum, die zum Unterlassen von Aktionen führe, weil man sich im Kamerafokus weniger traue $(03,04)$. Obwohl sie dies kurz feststellen, liegt der Schwerpunkt des Gesprächs auf einem ,permanenten Unwohlsein“ und Reflexionen über den aktiven Umgang damit.

\footnotetext{
11 Eine linksradikale Gruppe (02) bildet hier die Ausnahme. In dieser wird Videoüberwachung zwar als Bedrohung empfunden, die aber vor allem Aggressionen auslöse.
} 
Es werden auch zahlreiche Situationen benannt, in denen Videoüberwachung an Bedeutsamkeit und damit Abschreckungswirkung verliert, weil die Situationsdynamik die Aufmerksamkeit anders verteilt oder das Erreichen wichtiger Protestziele situativ maßgeblicher für Handlungsentscheidungen ist. Dazu gehören direkte Auseinandersetzungen mit Polizist/innen (03) oder Blockaden (03, 04). Zudem gehört die Präsenz von Polizei- und Medienkameras für einige $(01,03,04)$ bereits zum „Alltag auf Demos“ (04). Sehr explizit verneinen die Diskutant/innen der linken Jugendgruppe (04) abschreckende Wirkungen auf sich selbst. Der praktische Ungehorsam gegen die unterstellte polizeiliche Abschreckungsintention ist in diesem Fall wichtiger - als ein Moment der Selbstbehauptung und Bestätigung widerständiger Selbstbilder. Resistenz wird als Zeugnis der Zugehörigkeit zur internationalen linken Bewegung und gewissermaßen auch als solidarische Pflicht gegenüber sozialen Bewegungen in autoritären Staaten empfunden.

Abschreckung erweist sich somit in weitgehender Übereinstimmung mit dem medialen und juristischen Diskurs auch bei politischen Aktivist/innen verschiedenster Spektren als zentrales Deutungsmuster für Videoüberwachung. Es dient als Erklärung für ausbleibende Aktivitäten Anderer. Konkrete Erzählungen der Demonstrierenden legen jedoch nahe, dass Abschreckung durch Videoüberwachung nur unter besonderen Bedingungen tatsächlich auftritt. Vielmehr kommt es zu praktischen Adaptionen an die Videoüberwachung (Abschnitt 6). Abschreckung kann als positiv präventiv oder auch als repressiv gedeutet werden und spiegelt, wie im Folgenden auch anhand anderer Deutungen von Videoüberwachung gezeigt wird, die grundsätzlichen Beziehungen der Protestgruppen zur Polizei wider.

Die im Folgenden dargestellten Deutungsmuster sind enger an spezifische Versammlungssituationen gebunden, die im Grunde entweder als friedlich oder konflikthaft wahrgenommen werden und die Videoüberwachung jeweils in einem bestimmten Licht erscheinen lassen.

\subsection{Politische Repression}

Videoüberwachung wird insbesondere dann als ungerechtfertigte, willkürliche oder illegale Repression gedeutet, wenn die Einsatzsituation als friedlich bzw. konfliktarm wahrgenommen wird. Diese Situationsdefinition wird rhetorisch häufig durch die Nennung von Personengruppen hergestellt, die kollektivsymbolisch für Friedlichkeit stehen. In der liberalen und der Ultra-Gruppe $(01,05)$ steht die Überwachung von ,älteren Leuten“ und Familien sinnbildlich für einen unverhältnismäßigen Umgang mit friedlichen Demonstrierenden. Die rechten Demonstrierenden veranschaulichen ihre eigene Friedlichkeit und die Unmöglichkeit, sich gegen aggressive Gegendemonstrierende zu wehren, mit geschlechterstereotypen Zuschreibungen: sie betonen ostentativ, dass auch Frauen bei ihren Demonstrationen anwesend waren und durch Gegendemonstrierende verängstigt wurden (06). Die linken Diskussionsgruppen skandalisieren trotz einer prinzipiell kritischen Haltung zur Videoüberwachung vor allem solche Überwachungssituationen, in denen „friedliche“, „,bunte“ $(02,04)$ oder durch „lockere Reihen“ (03) gekennzeichnete Demonstrationen überwacht werden. Solchen als ungerechtfertigt empfundenen Überwachungssituationen stehen Situationen gegenüber, die, wie beispielsweise „Krawall“ oder der „Schwar- 
Tab. 3 Akzeptanz von Videoüberwachung in Abhängigkeit von politischer Strömungszugehörigkeit und Situationsdefinitionen

\begin{tabular}{|c|c|c|}
\hline \multirow{2}{*}{$\begin{array}{l}\text { Politische Grund- } \\
\text { strömungen }\end{array}$} & \multicolumn{2}{|c|}{ Situationsdefinition und dafür typische Personengruppen } \\
\hline & $\begin{array}{l}\text { Friedliche Situation } \rightarrow \\
\text { Videoüberwachung illegitim bzw. } \\
\text { illegal }\end{array}$ & $\begin{array}{l}\text { Konfliktsituation } \rightarrow \\
\text { Videoüberwachung akzeptiert }\end{array}$ \\
\hline $\begin{array}{l}\text { (Unpolitische) } \\
\text { Ultra-Fans (01) }\end{array}$ & $\begin{array}{l}\text { Trauermarsch, Choreografien, Fan- } \\
\text { märsche ohne Rivalitäten, Rauchen } \\
\text { im Stadion, teilweise Vermummung, } \\
\text { Kinder, Familien }\end{array}$ & $\begin{array}{l}\text { Aggression, Straftaten, Auftreten } \\
\text { rivalisierender Fangruppen }\end{array}$ \\
\hline $\begin{array}{l}\text { Linke Gruppen } \\
(02-04)\end{array}$ & $\begin{array}{l}\text { „Bunte“ Versammlung, „lockere Rei- } \\
\text { hen“, Sitzblockaden, ,ältere Leute“, } \\
\text { Kinder, Familien }\end{array}$ & $\begin{array}{l}\text { Auseinandersetzungen mit Polizei, } \\
\text { „Krawall“, rechte Ausschreitungen, } \\
\text { Schwarzer Block, geschlossene Rei- } \\
\text { hen, Straftaten }\end{array}$ \\
\hline Liberale (05) & $\begin{array}{l}\text { Eigene Demonstrationen, ausgelas- } \\
\text { senes Feiern beim Christopher Street } \\
\text { Day }\end{array}$ & $\begin{array}{l}\text { Rechter Protest mit Gegenprotest, zi- } \\
\text { viler Ungehorsam, Straftaten, Gewalt, } \\
\text { homophobe Angriffe }\end{array}$ \\
\hline Rechte (06) & $\begin{array}{l}\text { Eigene Demonstrationen, Frauen, } \\
\text { „Ältere“ }\end{array}$ & $\begin{array}{l}\text { Antifaschistischer Gegenprotest, Me- } \\
\text { dienanwesenheit, Straftaten }\end{array}$ \\
\hline
\end{tabular}

ze Block“, als Kollektivsymbole für Konflikt und Gefahr gelten und in denen die Demonstrierenden den Kameraeinsatz nachvollziehen können. Die situationsdefinierenden und damit Legitimität und Illegitimität der Überwachung begründenden Merkmale werden in ihrer Verteilung auf die jeweiligen politischen Strömungen in Tabelle 3 dargestellt. Mit diesen Kategorien beziehen sich alle Demonstrierenden auf Unterscheidungen, die auch für die Polizei maßgeblich sind (dazu Ullrich 2017). Die Selbstwahrnehmung und die Wahrnehmung des Handelns Anderer orientieren sich nicht an politischen Kategorien, sondern an polizeilichen Gefahrenkategorien. Die implizite Übernahme von polizeilichen kategorialen Ordnungssystemen durch die Protestierenden weist auf eine zumindest partielle Durchsetzung der polizeilichen Subjektivierungsform hin.

Dissens zur Polizei besteht in diesen Schilderungen also nicht auf der Ebene der leitenden Kategorien, sondern entfaltet sich immanent entlang der Frage ihrer angemessenen Anwendung. In friedlichen Situationen interpretieren die Demonstrierenden den Einsatz von Videoüberwachung hingegen im Deutungsmuster politische Repression. In diesem treten Kriminalisierung, Provokation und Datensammlung als Ausprägungen (Subtypen) auf.

\subsubsection{Kriminalisierung}

Alle linken und linksradikalen Gruppen fühlen sich durch Videoüberwachung und ein hohes Polizeiaufgebot bei Demonstrationen als Kriminelle diffamiert, insbesondere da die Kamera das „Signal nach außen“ vermittle, ,die Polizei muss die arme Bevölkerung davor schützen, vor diesen extremst gefährlichen Leuten“ (03). Aus Sicht der Betroffenen ist die Kamera für die Demonstration ein Stigma, das die Öffentlichkeit abschreckt. Diese (politische) Isolation der Versammlungen deuten sie als polizeiliche Intention. Dahinter vermuten sie ein Feindbild der Polizist/innen bzw. des Staates, die den Linken bzw. Linksradikalen oder gar jeder Massenan- 
sammlung feindlich gegenüberstünden bzw. diese als Gefahr ansähen (01-03). Die Selbstwahrnehmung als (politische) Aktivist/innen mit legitimen Zielsetzungen und die erfahrene symbolische Zuweisung einer Gefährder/innenrolle geraten so in Widerspruch. Die Polizei nehmen sie als politisch motivierten Akteur wahr, der nach außen die Botschaft des Protests entstelle, ihn damit entpolitisiere und stattdessen als Gefahr darstelle. ${ }^{12}$

Am deutlichsten tritt die Politisierung des polizeilichen Handelns zutage, wenn der Kameraeinsatz als Anzeichen rechter Einstellungen von Polizist/innen interpretiert wird (04). So überträgt die linke Jugendgruppe das für sie charakteristische Deutungsmuster einer Rechtslastigkeit der Polizei auf die Videoüberwachung. ${ }^{13}$ Auch die rechte Gruppe (06) vermutet für Situationen, in denen die Polizei gegen die eigene Protestgruppe vorgeht oder in denen sie die eigenen Versammlungen nicht gegen Gegenproteste durchsetzt, ein politisches Motiv für das Auftreten bzw. Unterbleiben polizeilicher Interventionen. Sie kritisieren entsprechend, dass die Polizei den Gegenprotest nicht immer ausreichend bekämpft oder filmt. Allerdings schreiben sie sich selbst, im Gegensatz zu den als aggressiv wahrgenommenen linken Gruppen, keine eigene Handlungsfähigkeit zu.

Zwischen der linken Jugendgruppe und der rechten Gruppe, die sich hinsichtlich der Protestformen, Situationsbeschreibungen und dem Empfinden einer ungerechten Behandlung der eigenen Gruppe ähneln, wird jedoch auch eine Differenz deutlich, die mit ihren politischen Grundpositionen zusammenhängt. So zielt die Argumentation der linken Jugendgruppe letztlich auf eine egalitäre Anwendung von Videoüberwachung und eine Übernahme von Eigenverantwortung für das Handeln auf Versammlungen. Die rechten Demonstrierenden hingegen nutzen eigene Unsicherheitserfahrungen, um Forderungen nach einem härteren polizeilichen Vorgehen ausschließlich gegen politische Gegner/innen zu legitimieren, deren Recht auf Gegenprotest in der Nähe rechter Versammlungen sie bestreiten. Die linksradikale Position kulminiert dagegen, im Sinne ihrer antietatistischen Grundposition und damit trotz der Übernahme polizeilicher Kategorien in den Situationsbewertungen, in einer grundsätzlichen Ablehnung von Videoüberwachung und Polizei.

\subsubsection{Provokation}

Ein weiteres bei linken Demonstrierenden und Ultras auftauchendes Deutungsmuster ist die Provokation durch Videoüberwachung. Es gehört zu einem breiten Spektrum als provozierend empfundener Verhaltensweisen der Polizei.

Filmen, Schikaniererei, in Form von irgendwelchen Sprüchen, Provokationen, [...] sicherlich gibt es auch immer zwei Seiten der Medaille, aber die Seite der Polizei auf der Medaille gibt es auf jeden Fall auch. [...] da kommt der Polizist und sagt: So, die Zecken bringen es nicht mehr, was ist heute mit euch? [...]

\footnotetext{
${ }_{12}$ Die Rechten erleben solche Positionierungszumutungen besonders durch die Medien, in denen sie als „Provokateure“ und nicht ihrem Selbstbild entsprechend als Verteidiger/innen des Rechtsstaats dargestellt würden.

${ }^{13}$ Dieses Deutungsmuster ist in antifaschistischen Protestspektren weit verbreitet, worauf der dort geläufige Slogan „Deutsche Polizisten schützen die Faschisten“ hindeutet.
} 
dann geht es los mit aus der zweiten Reihe Zuschlagen, dann hinter dem Mob [der Polizist/innen; d. A.] wieder verschwinden, und dann sagen sie alle: Da hat doch niemand zugeschlagen. (01)

Als Provokationen empfinden die Ultra-Fans polizeiliche Handlungen, die in friedlichen, aber auch bereits angespannten Situationen Aggressionen auslösen oder verstärken sollen und die sie als Anzeichen für die Feindseligkeit der Polizei bzw. der Polizist/innen interpretieren. Polizist/innen suchten die Eskalation und übten zu diesem Zweck grundlos Gewalt gegen Demonstrierende oder Fans aus. Die Videoüberwachung, die als willkürlicher, grundloser oder zum Teil illegaler Eingriff in das Versammlungsrecht gilt, setzt sonstigen polizeilichen Kontrollmaßnahmen aus dieser Sicht nur ein „Krönchen“ (02) oder ,i-Tüpfelchen“ (05) auf. Zudem frustriert die Demonstrierenden und die Ultra-Fans der mit Macht- und Sichtbarkeitsasymmetrien verbundene Mangel an effektiven Möglichkeiten, sich gegen polizeiliche Übergriffe (legal) zur Wehr zu setzen (vgl. Singelnstein 2014). Die eigene Aussage gelte vor Gericht weniger als die von Polizist/innen. Eine Anzeige sei aufgrund der Anonymität der Polizist/innen oft nicht möglich. Das wiederholte Auftreten von Situationen, in denen die Ultra-Fans sich nicht gegen als unrechtmäßig empfundenes polizeiliches Handeln wehren konnten, erfahren sie als Entwürdigung (01). Angesichts der erfahrenen Missachtung bis hin zur Verletzung ihrer körperlichen Integrität fühlen sie sich als „Dummy“ oder wie „Vieh“ behandelt. In eskalierenden Situationen erkennen sie zwar eine Beteiligung von Fans an, betonen aber die Rolle der Polizei. Dadurch grenzen sie solche Situationen deutlich von Ausschreitungen $\mathrm{ab}$, die von der eigenen Seite ausgingen.

Die Demonstrierenden der liberalen Gruppe erleben selbst auf Versammlungen keine derartigen Provokationen durch Polizist/innen. Doch auch sie bewerten die im Videostimulus der Gruppendiskussion gezeigten Szenen, in denen Polizist/innen innerhalb einer Demonstration stehen und filmen, als bewusste polizeiliche Provokation (05).

Polizeiliche Videoüberwachung nehmen alle Gruppen auch als Auslöser von Konflikten wahr, da sie aggressionssteigernd wirke (vgl. auch Ullrich 2011). Wie auch im Deutungsmuster der Kriminalisierung treten die durch die Videoüberwachung angezeigte polizeiliche Positionierung von Demonstrierenden als gefährliche Subjekte und deren Selbstwahrnehmung als politische Akteur/innen auseinander. Der Unterschied zur Kriminalisierung besteht darin, dass die Demonstrierenden der Polizei bzw. einzelnen Polizist/innen über die symbolisch durch die Videoüberwachung angezeigte Missachtung des politischen Anliegens von Demonstrationen hinaus das Motiv unterstellen, die Kriminalisierung gewissermaßen realisieren zu wollen, indem sie Protestierende bewusst zu Gewalt oder anderen Gesetzesverstößen verführen.

\subsubsection{Datensammlung}

Auch das Deutungsmuster der Datensammlung bezieht sich auf friedliche Situationen, in denen aus Sicht der Befragten kein legitimer Anlass für Videoüberwachung vorliegt und die Versammlung stattdessen genutzt werde, um Anwesende zu identifizieren $(01,03,04,06)$. Im Zusammenhang mit der Datensammlung verweisen 
die Diskussionsteilnehmer/innen auch auf andere Mittel des „Überwachungsstaates“ (05), die ihre Annahme bestätigen, dass es ein staatliches Interesse an der Sammlung von Daten über Protestierende gibt (z. B. werden Fan-Datenbanken, die biometrische Datenauswertung oder der Umgang mit der Opposition in Russland oder der DDR als vergleichbare Formen der Überwachung benannt). Wie weitgehend und folgenreich sie die Datensammlung einschätzen, hängt vom Vertrauen in den Rechtsstaat und von der Einschätzung der zukünftigen politischen Entwicklung ab. Diskussionsgruppen, die die Rechtsstaatlichkeit Deutschlands bzw. der Polizei grundsätzlich anzweifeln, befürchten eine langfristige Speicherung und spätere Nutzung von Videos, auch von solchen, die keine konkreten Straftaten dokumentieren (01-03). Für die rechten Demonstrierenden kommt die Datensammlung dem Eintritt der eigentlich positiv besetzten Polizei in einen „Dreifrontenkrieg“(06) an der Seite von Antifa und Medien gleich, gegen die sie sich bereits in einem ,Zweifrontenkrieg“ befänden. Wenn jedoch ein prinzipielles Rechtsstaatsvertrauen vorhanden ist, gehen die Demonstrierenden eher davon aus, dass die Polizei die gespeicherten Daten löscht. Diese Argumentation verdichtet sich - bei Erfüllung der normativen Rolle der Polizei als neutraler Schiedsrichter - in der Annahme, dass, wer keine Straftaten begehe, auch keine negativen Folgen zu befürchten habe (04). Jedoch erscheint die ab 2014 erlebte Polarisierung der Gesellschaft sowohl der rechten Gruppe als auch der linken Jugendgruppe als ein Grund, sich auf eine mögliche Neubewertung der Videoüberwachung einzustellen, da beide Gruppen eine negative Entwicklung der Rechtsstaatlichkeit und damit verbunden eine Ausweitung der politischen Repression befürchten. Die befürchtete Verschiebung der politischen Ordnung impliziert für sie daher auch eine Neubestimmung ihrer Positionierung zu dieser Ordnung und gegenüber der Polizei. Die mit dem Medium Videokamera verbundene Ausweitung von Raum- und Zeitbezügen des Handelns bewirkt, wie diese Deutungen zeigen, dass die Subjektivierungsweisen nicht nur auf je aktuelle gesellschaftliche und politische Ordnungen sowie deren Umgang mit Abweichung und Protest bezogen sind, sondern auch auf zukünftige.

\subsection{Akzeptierte Normalität im Konflikt}

Obwohl in allen Gruppen negative Bewertungen auftreten und in den linken Gruppen auch deutlich dominieren, sehen sie Videoüberwachung in Konfliktsituationen durchaus als normales Konfliktmittel an (02-04). Auch diese Deutungen sind mit bestimmten wiederkehrenden Situationen bei Demonstrationen verknüpft. Ultra-Fans und linke Demonstrierende sehen in Videoüberwachung zwar keinen Schutz, finden sich jedoch mit ihrem Einsatz in Auseinandersetzungen mitunter ab. Vor allem kontrastierende Beschreibungen verdeutlichen die Unterscheidung von Einsatzgründen, die sie als gerechtfertigt anerkennen, und solchen, die sie verurteilen. Akzeptierte Einsatzgründe sind Steinwürfe, körperliche Auseinandersetzungen oder die Formierung eines Schwarzen Blocks. In den Darstellungen wird die Handlungskette „Straftat/aggressives Verhalten - Videoüberwachung - gezielte Festnahme“ durchaus als legitim nachvollzogen. Videoüberwachung gilt dann vor allem als Beweismittel.

Trotz dieser situativen Akzeptanz der Videoüberwachung als normales Einsatzmittel der Polizei erhoffen sich die Demonstrierenden davon keine Vorteile. Statt- 
dessen bringt die mit der Videoüberwachung verbundene technische Ausweitung polizeilicher Kontrollmöglichkeiten für sie vor allem Nachteile und Handlungsunsicherheiten mit sich. So beobachten linksradikale Demonstrierende die Verlagerung von Strafverfolgungsmaßnahmen auf Basis angefertigter Videobilder in den Zeitraum nach der jeweiligen Versammlung. Obwohl mit dieser Ausweitung des polizeilichen Handlungsspielraums Verunsicherungen verbunden sind, verfolgen die Reflexionen nicht den Zweck, dem Protest fernzubleiben, sondern zielen auf Verhaltensanpassungen und Sicherheitspraktiken.

\subsection{Schutz}

Die liberalen (05) und die rechten Demonstrierenden (06) deuten Videoüberwachung auch als Schutz vor Angriffen. Sie gehen dabei von einer Abschreckungswirkung aus oder davon, dass die Videoaufnahmen der Strafverfolgung nutzen. Der „Videoschutz" wehrt nicht nur politische Gegner/innen ab, sondern dokumentiert auch unrechtmäßige Amtshandlungen, wie die rechte Gruppe argumentiert. Der Schutz gilt aber nicht nur den Demonstrierenden, sondern auch den Polizist/innen, die durch das Videomaterial ungerechtfertigte Beschuldigungen zurückweisen können. ${ }^{14}$ Die rechte und die liberale Gruppe verbindet dabei, dass sie ihre Demonstrationen als durch politische Gegner/innen gefährdet wahrnehmen und die Polizei als Beschützerin ihrer Versammlungen gilt. Diese Diskussionsgruppen drücken ein gewisses Maß an Eigeninteresse an der Videoüberwachung aus (vgl. auch Knopp und Ullrich 2016). Sie bewerten die polizeiliche Videoüberwachung in den meisten im Alltag erlebten Versammlungssituationen positiv. Die rechten Demonstrierenden fordern auch mehr Videoüberwachung gegen politische Gegner/innen. Die liberalen Demonstrierenden schätzen die Videoüberwachung als möglichen Schutz vor Angriffen auf ihre Versammlungen und als Mittel der Strafverfolgung. Da von dieser Gruppe aber eine polizeiliche Videoüberwachung auf Demonstrationen kaum beobachtet wird, beziehen sich ihre Erfahrungen vor allem auf die stationäre Videoüberwachung und auf alltägliche Begebenheiten.

In den dargestellten Deutungsmustern tritt eine Überlagerung verschiedener Logiken zutage. Die Deutungen der Diskussionsteilnehmer/innen changieren zwischen einer politischen Logik und einer Sicherheitslogik. Der Sicherheitslogik folgend deuten die Demonstrierenden Situationen und Handlungen entlang des Gegensatzpaares Sicherheit - Gefahr. Die politische Deutungslogik verläuft entlang des Gegensatzpaares Unterdrückung - Beförderung der Sichtbarkeit einer politischen Position im öffentlichen Raum und vermutet hinter polizeilichen Handlungen ein solches politisches Motiv.

Die ambivalenten Deutungsmuster artikulieren einen Subjektivierungseffekt polizeilicher Kontrollpraktiken, da die Demonstrierenden bei ihren Deutungen auf die in Polizeimaßnahmen praktisch umgesetzten und für die Demonstrierenden sichtbar angezeigten Kategoriensysteme zurückgreifen und ihr Handeln und das Han-

\footnotetext{
${ }^{14}$ Die linken Gruppen und die Ultra-Gruppe, die alle von unverhältnismäßiger Polizeigewalt berichten, sprechen dagegen davon, dass gerade illegales Polizeihandeln nicht dokumentiert werde und die Polizei gegen Demonstrierende, die selbst filmen, aggressiv vorgehe (01-04).
} 
deln anderer zumindest teilweise anhand dieser Kategorien deuten. Auch kollektive Handlungsmuster der Demonstrierenden orientieren sich an der Polizei, indem sie gemeinsam mit ihr oder gegen sie Sicherheitspraktiken etablieren.

\section{Sicherheitskulturen: Protestpraktiken im Spannungsverhältnis von Sicherheit und politischer Handlungslogik}

[Dass man sich] auf jeden Fall klarer machen sollte - wobei man nicht in die Paranoidität des [mit ängstlicher Stimmlage] Oh-nein-ich-kann-nichts-mehr-machen verfallen sollte [...] - wann ist hier gerade irgendwie was richtig kacke und wann muss ich mich irgendwie anders verhalten. (03)

Die von dem zitierten Demonstrierenden eingeforderte Selbstreflexion benennt ein praktisches Problem, das sich auf verschiedene Weise allen Protestierenden stellt: Sicherheit für das eigene Handeln und kollektive Protestformen herzustellen und dabei gleichzeitig gegenüber der Öffentlichkeit als intelligible politische Subjekte mit legitimen Forderungen aufzutreten und damit (auf Dauer) politisch handlungsfähig zu sein.

Wie die verschiedenen Protestspektren und -gruppen diese oft widersprüchlichen Ansprüche austarieren, ist ein Aspekt der jeweiligen Protestkulturen, den wir Sicherheitskultur nennen. Unter Sicherheitskulturen verstehen wir die Gefüge aus protestspektrenspezifischen Praktiken, darauf bezogenen Normen und kollektivem Wissen, die sinnhaft auf die Absicherung von politischem Engagement im Kontext wahrgenommener Gefahren bezogen sind. ${ }^{15}$ Sie umfassen Sicherheitspraktiken, Bewegungsdiskurse über den Umgang mit Polizei und Justiz - inklusive einer umfangreichen Ratgeberliteratur -, spezielle Trainings, in denen Protestpraktiken eingeübt werden, Artefakte und Medien, wie z. B. Vermummungsgegenstände, Videokameras oder Funkgeräte, sowie normative Vorstellungen über Umgangsweisen mit Gefahren. Die sicherheitsbezogenen Diskurse und Praktiken sind in der Praxis eng mit unterschiedlichen Protestkulturen verwoben. Sie prägen die Art und Weise, wie bestimmte Protestspektren Demonstrationen planen und abhalten, wie Demonstrierende kommunizieren und sich öffentlich präsentieren. Das Konzept der Sicherheitskulturen verdeutlicht analytisch den Anteil der Polizei und anderer erwarteter Gefahren an den Sinngebungen von Protestpraktiken. ${ }^{16} \mathrm{Zu}$ den als Gefahren eingeschätzten Phänomenen können, wie der Fall der rechten Demonstrierenden (06) zeigt, auch Medienvertreter/innen oder politische Gegner/innen gehören.

Der Begriff der Sicherheitskultur weist damit über den engeren Gegenstandsbereich der Videoüberwachung hinaus, da er alle sicherheitsbezogenen Praktiken

\footnotetext{
15 Den Begriff „Sicherheitskulturen“ übernehmen wir von Starr et al. (2008), die ihn ohne nähere Definition zur Kennzeichnung von organisationsspezifischen Umgangsweisen mit staatlicher Überwachung verwenden.

16 Da nicht alle Protestspektren die Polizei selbst als Unsicherheitsfaktor ansehen, sondern manche Spektren sie als Teil der Herstellung von Handlungssicherheit erachten, wird verständlich, dass es sich bei den Sicherheitskulturen nicht immer nur um Antirepressionskulturen handelt, die für die autonome und radikale Linke, aber auch neonazistische Gruppierungen charakteristisch sind.
} 
erfasst und nicht bloß Gegenüberwachungspraktiken, die an der Videoüberwachung orientiert sind. Zugleich verdeutlicht der Begriff ein Spezifikum der Reaktionsweisen von Protestierenden auf Videoüberwachung, das bei der stationären Videoüberwachung des öffentlichen Raums kaum auftritt: Die Sicherheitspraktiken besitzen einen kollektiven Charakter und können mitunter nur zusammen mit anderen Protestierenden ausgeführt werden. Die jeweiligen Protestspektren verbreiten sie und verhandeln ihre Ausgestaltung. Sie geben Wissen und Fertigkeiten organisiert weiter. Darüber hinaus bilden Sicherheitspraktiken wichtige Elemente des Selbstverständnisses von Protestspektren, insofern sie Widerstand, Trotz oder auch Kooperation ausdrücken (siehe auch Abschnitt 5.1).

Sicherheitspraktiken, die sich speziell gegen die Videoüberwachung richten, kommen nur in den stärker von Videoüberwachung betroffenen Diskussionsgruppen, also bei den Linken und den Ultra-Fans explizit zur Sprache. Die stärkere Exposition dieser Gruppen geht mit einer stärkeren Auseinandersetzung mit Videoüberwachung einher. Diese Gruppen machen deutlich, dass die Demonstrierenden ihr Handeln stark an der Polizei und ihren (Video-)Überwachungspotenzialen ausrichten und das Verhältnis zur Polizei und den von ihr ausgedrückten Verhaltenserwartungen umfangreich reflektieren. Die rechten und liberalen Gruppen kommen dagegen auf ihren Demonstrationen selten bis nie mit Videoüberwachung in Berührung. Ihre videoüberwachungsbezogenen Reflexionen sind entsprechend weniger ausführlich, und sie sprechen kaum über Gegenüberwachung. Die Polizei ist in diesen Gruppen eher Teil von Absicherungsarrangements.

Auf der Grundlage der Gruppendiskussionen lassen sich im Hinblick auf Videoüberwachung fünf Sicherheitskulturen unterscheiden: reflektierte Militanz, Überwachungsrealismus, kritische Bürger/innen, Kooperation und Passivität.

\subsection{Reflektierte Militanz}

Die Sicherheitskultur der reflektierten Militanz ist an einer Idealvorstellung von Protest orientiert, die beispielhaft im einleitenden Zitat zu diesem Abschnitt zum Ausdruck kommt und der es um das Erreichen von Protestzielen bei umfangreicher Absicherung gegenüber Videoüberwachung durch Mittel des kollektiven Selbstschutzes geht. Auch illegale Sicherheitspraktiken sehen die Protestierenden situativ als sinnvollen Schutz an. Zudem gelten diese als ein politischer Ausdruck des Widerstands gegen die Übermacht der Polizei. Diese Opposition soll mitunter auch gegenüber der Öffentlichkeit dargestellt werden. Somit stellt sich in dieser Sicherheitskultur eine gewisse Konvergenz zwischen politischen und sicherheitsbezogenen Orientierungen ein. In den Diskussionen jener Gruppen, die dieser Sicherheitskultur folgen $(02,03)$, ist eine weitgehende Rationalisierung der Sicherheitspraktiken Ziel der umfangreichen Reflexionen, um mit der im linksradikalen Protestspektrum wahrgenommenen „Paranoia“ umzugehen und dauerhaft, z. T. auch militant handlungsfähig zu bleiben. Die Versuche, das Protesthandeln zu rationalisieren, sind in der Praxis jedoch nur beschränkt umsetzbar, denn immer wieder rücken situative Zwänge und Dynamiken in den Vordergrund (z.B. unvorhergesehene Auseinandersetzungen, plötzliche Festnahmen). Zu den charakteristischen Artefakten dieser Sicherheitskultur gehören Mittel der Vermummung und schwarze Kleidung. Das Bedürfnis nach Anonymität 
gegenüber der Polizei besteht auch ohne den Kontext einer konkreten Konfliktsituation oder militanter Aktionsformen. Insofern die Demonstrierenden hohe Sanktionsrisiken aufgrund der Vermummung wahrnehmen, greifen sie auch auf andere Anonymisierungspraktiken zurück ${ }^{17}$ oder verzichten gänzlich auf solche Mittel und ignorieren die Videoüberwachung bewusst. Die Demonstrierenden beobachten mehr oder minder systematisch das Verhalten von Polizist/innen und suchen nach Kameras, an deren Anwesenheit und Fokussierung auf bestimmte Personen sie polizeiliche Klassifizierungen und drohende repressive Maßnahmen ablesen. Daraufhin passen sie ihr Handeln an. Das Wissen um die Funktionsweise der Videoüberwachung befähigt die Demonstrierenden somit auch zu ihrer Umgehung und zu widerständigen Reaktionen.

\section{2 Überwachungsrealismus - den Blicken genügen und widerstehen}

Diese Sicherheitskultur ist davon geprägt, dass die Protestierenden verschiedene Sicherheitspraktiken, die Anonymität herstellen sollen, und ihre präferierten Ausdrucksformen und Zielvorstellungen als schwer auflösbaren Widerspruch erleben. Im Vordergrund steht für die Demonstrierenden dabei die Darstellung der politischen Ziele des Protests, die an weit verbreiten Vorstellungen über das legitime Erscheinungsbild von „,bunten“ Versammlungen orientiert ist $(02,04)$, bzw. die Darbietung von Fankultur und Unterstützung des eigenen Fußballvereins (01). Die politischen Gruppen in dieser Sicherheitskultur definieren ihre Protestziele primär dadurch, dass möglichst viele Menschen an Demonstrationen und Blockaden teilnehmen. Vermummung oder uniforme schwarze Kleidung, wie sie das Negativimage des Schwarzen Blocks prägen, gelten als problematisch, da sie auf das Publikum abschreckend wirken und polizeiliche Interventionen auslösen könnten. Die linke Jugendgruppe kritisiert dabei vor allem das ausschließende Auftreten des Schwarzen Blocks. Trotzdem erkennen sie Vermummung und schwarze Kleidung als Maßnahmen gegen Videoüberwachung und vor allem gegen Bildaufnahmen durch Rechte durchaus an und wenden sie z. T. selbst an. Sie reflektieren das aber kritisch: „Dadurch, dass sich die Leute dann vermummen, hat das einen viel krasseren Eindruck [...], weil sie [andere Jugendliche; d. A.] einfach sagen: Was soll denn das? Warum ziehen die [Demonstrierenden; d. A.] sich so an? Weil sie es einfach nicht verstehen und es einfach auch ein bisschen als Gefahr sehen“ (04). Es ergebe „ein ganz anderes Erscheinungsbild, wenn die Demonstranten nicht nur in einer einheitlich dunklen Farbe auf der Demo sind, sondern in ganz normaler Alltagskleidung oder [...] bunteren Farben“ (04). Die Kritik, dass die Homogenisierung durch Vermummung und schwarze Kleidung abschrecke, folgt einem Idealbild von pluralistischem Protest. Pluralität auch visuell darzustellen, ermögliche es, auch das Publikum für den Protest zu gewinnen. Die Orientierung der Protest- und Sicherheitspraktiken an diesen weit verbreiteten Vorstellungen über legitime Protestformen stellt das ,realistische“ Moment dieses Protestspektrums dar - im Gegensatz zur Darstellung einer radikalen Ablehnung der politischen Darstellungsnormen, wie dies in der Sicherheitskultur der

${ }_{17}$ Genannt werden z.B. Wegdrehen, die Hand vor das Gesicht halten, überwachte Bereiche umgehen oder ausweichen. Beschwerden bei Polizist/innen werden nicht erwogen oder als aussichtslos eingeschätzt. 
reflektierten Militanz auftritt. Dennoch sehen sich auch die Protestierenden dieser Sicherheitskultur Unsicherheiten und Gefahren ausgesetzt. Sie weichen aber auf als weniger aggressiv wahrgenommene - wenn auch als weniger effektiv angesehene - Gegenüberwachungspraktiken aus, darunter das Wegdrehen von Polizeikameras oder das Tragen von Brillen, Kopfbedeckungen und Schals, die das Gesicht teilweise verdecken.

\subsection{Kritische Bürger/innen}

Die Sicherheitskultur der kritischen Bürger/innen, die in unserem Sample innerhalb der liberalen Diskussionsgruppe (05) vertreten ist, zeichnet sich durch einen Umgang mit Überwachung und polizeilichen Maßnahmen aus, der als charakteristisch für das Verhältnis von kritischer Zivilgesellschaft und Staat gelten kann. Zwar bringen die Angehörigen dieser Gruppe der Polizei und dem Rechtsstaat prinzipiell Vertrauen entgegen; wenn jedoch Verletzungen des Versammlungsrechts oder eine extensive Überwachung auftreten würden, wäre man bereit, sich dagegen innerhalb des gesetzlichen Rahmens, z.B. durch Beschwerden oder auf dem Klageweg, zu wehren: ,,...] wäre eine unserer Demos so abgelaufen, dass ich das Gefühl habe, die dringen da permanent ein, dann hätte ich mich drüber unterhalten mit der Polizei“ (05). Einzelne Teilnehmer/innen der liberalen Diskussionsgruppe üben deswegen Sousveillance-Praktiken wie z. B. Polizeibeobachtungen aus.

\subsection{Kooperation: gegenseitige Unterstützung und Self-Policing}

Da die liberalen Demonstrierenden jedoch überwiegend keine Erfahrungen mit polizeilicher Videoüberwachung auf eigenen Versammlungen gemacht haben, berichten sie auch nicht über praktische Umgangsweisen mit ihr. Die Polizei sehen sie vor allem als Unterstützer an, obwohl auch festgestellt wird, dass diese Position nicht selbstverständlich ist. Sie schätzen ein, dass ,die Gefährdung, die von außen kommt, also die Homo-Demo angegriffen wird von Homophoben, dass dann die Polizei eigentlich diese Sicherheitsfunktion hat oder Schutzfunktion für die Demo und nicht wie hier [im Videostimulus für die Diskussion; d. A.], wo ja eher die Polizei eine Art, ja sowas ganz komisches, so ein Gefährdungspotenzial darstellt“ (05).

In der kooperativen Sicherheitskultur ist das praktische Verhältnis zur Polizei durch gegenseitige Unterstützung geprägt. Während die Polizei durch ein ermöglichendes Verhalten den Protest schützt, organisieren die Protestierenden selbst eine Self-Policing-Struktur, die stark auf Sicherheitsaspekte ausgerichtet ist: ,Zu Anfang lernt man sich mit der Polizei kennen [...], man spricht kurz miteinander, jeder weiß, welche Aufgabe er hat. Bei uns ist es so, wir haben immer Funkstrecken, wir alle sind auch untereinander verbunden, also auch unsere Fahrer in den Autos, wir hören uns quasi auf zwei Kanälen die ganze Zeit“"(05). Die Praktiken des SelfPolicing unterliegen einer stetigen Optimierung. Obwohl es nicht zu polizeilichen Eingriffen kommt, wolle man auch kleine Regelübertretungen vorauseilend unterbinden. Ein solcherart betont kooperatives und auf Prävention von Regelübertretungen ausgerichtetes Agieren entspricht den von der Polizei an die Protestierenden herangetragenen Erwartungen und trägt damit auch dazu bei, die Videoüberwachung von 
Demonstrationen zu verhindern, auch wenn dies nicht die primäre Orientierung in dieser Sicherheitskultur ist.

\subsection{Passivität}

Die Sicherheitskultur der rechten Demonstrierenden (06) ist im Versammlungsgeschehen und in Bezug auf Videoüberwachung beinahe durchgehend von einer Haltung der Passivität gekennzeichnet, die mit einem betont friedlichen Selbstbild korrespondiert. Die entsprechende Sicherheitskultur beruht auf einem im Grundsatz guten Verhältnis zur Polizei. Wenn die Polizei aber nicht als Beschützer in Aktion tritt, fühlen sie sich schutzlos den Medien, die kritisch über sie berichten, und den linken Gegendemonstrierenden ausgesetzt. Der Polizei kommt hier die Rolle zu, die politische Handlungsfähigkeit herzustellen: ,[...] die Polizeibeamten stehen normalerweise mit dem Rücken zu uns und wissen, dass sie von uns nicht angegriffen werden, und wir wiederum wissen, dass, wenn die Polizei bei vielen unserer Kundgebungen oder Demonstrationen nicht präsent wäre, dass wir wirklich tätlich angegriffen würden“ (06). Eigene praktische Reaktionen auf Gegendemonstrierende, Medien oder als ungerechtfertigt empfundene Polizeihandlungen kommen in der rechten Gruppendiskussion kaum zur Sprache.

\section{Fazit}

Die angesichts langjähriger gesellschaftlicher Debatten über die Überwachungsgesellschaft verbreitete und intuitiv einleuchtende, aus soziologischer Sicht jedoch zu simple und bis dato empirisch ungeprüfte These, dass polizeiliche Videoüberwachung von Demonstrationen eine abschreckende Wirkung hat und Aktivist/innen sich wegen der Überwachung aus der politischen Partizipation zurückzögen, kann nicht ohne Weiteres aufrechterhalten werden. Die befragten Aktivist/innen berichten zwar auch von Abschreckungswirkungen, jedoch vornehmlich in vagen Erzählungen über Dritte und als abstrakte Reverenz an den überwachungskritischen Diskurs - kaum jedoch als eigenes Erleben. Mit einem subjektivierungsanalytischen Blick kommen aber komplexe Machteffekte des im gegenwärtigen, selektiven Protest Policing prominenten Einsatzmittels Videoüberwachung auf die Welt- und Selbstverhältnisse und kollektiven Praktiken der Protestierenden in den Blick. Die Analyse des Zusammenspiels der Subjektformierung durch die polizeilichen Videokameras mit den Subjektivierungsweisen der Protestierenden, also den Selbstwahrnehmungen, -deutungen und den damit verbundenen Praktiken, macht diese Effekte sichtbar. Wie Videoüberwachung auf kamerabezogene Deutungsmuster (a) und Protestkulturen (b) wirkt, hängt von verschiedenen Bedingungen ab.

(a) Wichtig für die Analyseebene der Deutungsmuster ist, erstens, die Kameraexposition. Während allgemeine Reflexionen zur Thematik sowie eine Verunsicherung in der Überwachungssituation bei allen betroffenen Demonstrierenden anzutreffen sind, ist die Auseinandersetzung mit dem Thema besonders intensiv und elaboriert bei denjenigen, die regelmäßig mit eingreifenden Polizeimaßnahmen, darunter Videoaufnahmen, konfrontiert sind. Die Deutungsmuster von Videoüberwachung sind, 
zweitens, nur in ihrer Einbettung in die Staats- und Polizeibilder der Protestierenden verstehbar. Die Videoüberwachung bestätigt quasi die grundlegenden Beziehungen der Protestierenden zur staatlichen Ordnung, die auf den Demonstrationen durch die Polizei repräsentiert wird. Das zeigt sich insbesondere in den Deutungen der polizeilichen Motivation zu videografieren, die vom grundsätzlichen Vertrauen in die Polizei als Institution abhängen. Drittens sind auch situationsbezogene Deutungen und Bewertungen von Videoüberwachung zu verzeichnen. Je nach Situation wird der Einsatz der Kamera als politische Repression verstanden oder als nachvollziehbar bzw. sogar angemessen betrachtet. Die zumindest situative Akzeptanz für Videoüberwachung setzt aber voraus, dass sie sich nur gegen Personen richtet, deren Beobachtung als legitim erachtet wird, insbesondere tatsächliche „Störer“ oder Gegner/innen der eigenen Gruppe. Sie tritt also dann auf, wenn die durch die Kamera angezeigte polizeiliche Positionierung der Demonstrierenden als Gefährder/ innen mit der Selbstwahrnehmung oder der Wahrnehmung des Handelns Anderer korrespondiert.

Das bedeutet, dass die von Überwachung Betroffenen, trotz ihres weitgehend kritischen Verhältnisses zu Videoüberwachung, durch die Techniken der Subjektformierung lernen, den überwachenden Blick und die ihm zugrundeliegenden Kategorien nachzuvollziehen und zum Teil selbst anzuwenden. Die Verinnerlichung von polizeilichen Kategorien geht dabei über eine einfache Erfüllung von Erwartungen hinaus, da sich auch kritische Äußerungen oft der gleichen Kategorien (bei eigenen Bewertungen der Situation) bedienen. In Situationen, in denen die Protestierenden eine Abweichung der Überwachung von rechtlichen und polizeilichen Normen feststellen, empfinden sie diese Überwachung besonders stark als ungerechtfertigt, kriminalisierend und entpolitisierend. Daraus resultieren Ohnmachtsgefühle und Aggressionen sowie praktische Gegenreaktionen, die den Konflikt mit der Polizei verstärken können.

(b) In dem beschriebenen Spannungsverhältnis von Subjektivierungsform und individuellem wie kollektivem Selbstbild entstehen unterschiedliche Demonstrationspraktiken, mit denen Protestierende u.a. auf die unerwünschte Kamerapräsenz reagieren. Sie bleiben dem Protest nicht vorrangig fern oder geben ihr politisches Engagement auf, ${ }^{18}$ sondern setzen sich aktiv mit der Situation der Überwachung auseinander. Die Kameras bedingen zunächst Reflexionen, die die durch Überwachung ausgelöste Unsicherheit aufnehmen und Handlungssicherheit wiederherstellen sollen. In diesen Reflexionen geht es darum, in der Situation wahrgenommener Bedrohung durch die Polizei und ihre Kameras oder durch Dritte sowohl die eigene Sicherheit zu wahren (das heißt vor allem Anonymität und Schutz vor Repression) als auch die eigene politische Botschaft gegenüber der Öffentlichkeit sichtbar zu machen. Die protestspektrenspezifischen Sicherheitskulturen kollektivieren und institutionalisieren diesen Umgang mit der Verunsicherung. Sie zeigen oftmals die Zerrissenheit der von Videoüberwachung betroffenen Protestierenden im Verhältnis

\footnotetext{
18 An dieser Stelle muss aber darauf hingewiesen werden, dass ein Selektionseffekt unseres Samplings nicht ausgeschlossen werden kann: Personen, die durch Kameras von einer Demonstrationsteilnahme oder einem Aktivismus generell abgeschreckt worden sein könnten, waren nicht im Sample vertreten. Sie sind auf dem von uns gewählten Weg schlicht nicht zu rekrutieren.
} 
zu sich selbst und anderen angesichts des für viele Sicherheitskulturen konstitutiven Spannungsverhältnisses von Sicherheit und politischer Darstellung.

Gerade die widerständigen Praktiken verweisen aber auch auf ermächtigende Subjektivierungseffekte, denn Demonstrierende können, insofern sie das Funktionieren der Machttechnik Videoüberwachung erlernt haben, durchaus deren Abläufe nachvollziehen und dadurch auch umgehen. Zudem bilden widerständige Praktiken in manchen Sicherheitskulturen Identifikationspunkte für Vergemeinschaftungsprozesse, indem sie Widerstandsgeist und Trotz darstellen.

Die Sicherheitskulturen und ihre zwei Referenzpunkte „eigene Sicherheit“ und ,politische Handlungsfähigkeit“" strukturieren die videoüberwachungsbezogenen Erfahrungen und Praktiken von Demonstrierenden. Durch diese praktische Verarbeitung wird Videoüberwachung Anlass für das Entstehen neuer und die Veränderung bestehender Protestformen sowie in manchen Protestspektren für spezialisierte Diskurse. In allen Gruppen treten solche Praktiken der Selbstkontrolle auf, die in den linken Gruppen und bei den Ultra-Fans auch dezidiert auf Videoüberwachung bezogen sind. Diese zielen aber eher auf die Bewahrung und Stärkung der protestgruppenspezifischen politischen Handlungsfähigkeit denn auf eine einfache Anpassung an polizeiliche Normvorstellungen. Somit entstehen Protestkulturen nicht zuletzt aus der Interaktion mit der Polizei und polizeilicher Überwachung.

Trotz der Relativierung der Abschreckungsthese bleibt die polizeiliche Videoüberwachung aus demokratietheoretischer, insbesondere grundrechtlicher Perspektive also von Relevanz. Verschiedene Demonstrierende fühlen sich durch Videoüberwachung verunsichert, kriminalisiert und stigmatisiert. Ihre politische Subjektivität und ihre Protestpraktiken bilden sich im Kontext der polizeilichen Subjektivierungsformen aus. Daher ist Protest eben nicht nur ,ein Stück ursprünglich-ungebändigter unmittelbarer Demokratie“ (BVerfGE 69, 315), sondern auch Resultat polizeilicher Macht.

Danksagung Der Aufsatz entstand im Rahmen des Forschungsprojekts „Videoüberwachung von Versammlungen und Demonstrationen. Praxis und Wissensformen von Polizei und Demonstrierenden (ViDemo)“, gefördert von der Deutschen Forschungsgemeinschaft (GZ: UL 389/3-1). Wir danken den Teilnehmer/innen des ipb-Kolloquiums „Politik von unten“ für die kritische Diskussion.

Open Access Dieser Artikel wird unter der Creative Commons Namensnennung 4.0 International Lizenz (http://creativecommons.org/licenses/by/4.0/deed.de) veröffentlicht, welche die Nutzung, Vervielfältigung, Bearbeitung, Verbreitung und Wiedergabe in jeglichem Medium und Format erlaubt, sofern Sie den/die ursprünglichen Autor(en) und die Quelle ordnungsgemäß nennen, einen Link zur Creative Commons Lizenz beifügen und angeben, ob Änderungen vorgenommen wurden.

Funding Open access funding provided by University of Vienna.

\section{Literatur}

Arzt, C., \& Ullrich, P. (2016). Versammlungsfreiheit versus polizeiliche Kontroll- und Überwachungspraxis. vorgänge. Zeitschrift für Bürgerrechte und Gesellschaftspolitik, 55(1), 46-60.

Baumgarten, B., \& Ullrich, P. (2016). Discourse, power, and governmentality. Social movement research with and beyond Foucault. In J. Roose \& H. Dietz (Hrsg.), Social theory and social movements. Mutual inspirations (S. 13-38). Wiesbaden: Springer VS. 
Bernhardt, H. (2014). Die „einschließende Absperrung“ bei Demonstrationen. Rechtliche Aspekte, psychologische Wirkungszusammenhänge und taktische Erfordernisse. Hilden: Verlag Deutsche Polizeiliteratur.

Bührmann, A. (2004). Der Kampf um „weibliche Individualität“. Zur Transformation moderner Subjektivierungsweisen in Deutschland um 1900. Münster: Westfälisches Dampfboot.

Bührmann, A. (2012). Das unternehmerische Selbst. Subjektivierungsform oder Subjektivierungsweise? In R. Keller, W. Schneider \& W. Viehöver (Hrsg.), Diskurs - Macht - Subjekt. Theorie und Empirie von Subjektivierung in der Diskursforschung (S. 145-164). Wiesbaden: VS Verlag für Sozialwissenschaften.

Death, C. (2010). Counter-conducts. A Foucauldian analytics of protest. Social Movement Studies, 9, 235-251.

Deleuze, G. (1993). Postskriptum über die Kontrollgesellschaften. In G. Deleuze, Unterhandlungen. 1972-1990 (S. 254-262). Frankfurt a. M.: Suhrkamp.

Dießelmann, A.-L. (2015). Ausnahmezustand im Sicherheits- und Krisendiskurs. Eine diskurstheoretische Studie mit Fallanalysen. Siegen: universi.

Feeley, M. M., \& Simon, J. (1992). The new penology. Notes on the emerging strategy of corrections and its implications. Criminology, 30, 449-474.

Feest, J., \& Blankenburg, E. (1972). Die Definitionsmacht der Polizei. Strategien der Strafverfolgung und soziale Selektion. Düsseldorf: Bertelsmann Universitätsverlag.

Foucault, M. (1994). Überwachen und Strafen. Die Geburt des Gefängnisses. Frankfurt a. M.: Suhrkamp.

Foucault, M. (2005). Subjekt und Macht. In M. Foucault, Analytik der Macht (S. 240-264). Frankfurt a. M.: Suhrkamp.

Gillham, P. F. (2011). Securitizing America. Strategic incapacitation and the policing of protest since the 11 September 2001 terrorist attacks. Sociology Compass, 5, 636-652.

Haggerty, K. D., \& Ericson, R. V. (2000). The surveillant assemblage. British Journal of Sociology, 51, $605-622$.

Heim, T. (2013). Metamorphosen des Kapitals. Kapitalistische Vergesellschaftung und Perspektiven einer kritischen Sozialwissenschaft nach Marx, Foucault und Bourdieu. Bielefeld: Transcript.

Höffling, C., Plaß, C., \& Schetsche, M. (2002). Deutungsmusteranalyse in der kriminologischen Forschung. Forum Qualitative Sozialforschung, 3(1), Art. 14.

Kitzinger, J. (1994). The methodology of focus groups. The importance of interaction between research participants. Sociology of Health \& Illnes, 16, 103-121.

Klauser, F. R. (2006). Die Videoüberwachung öffentlicher Räume. Zur Ambivalenz eines Instruments sozialer Kontrolle. Frankfurt a. M.: Campus.

Knopp, P., \& Ullrich, P. (2016). Kampf um die Bilder. Videoüberwachung und Gegenüberwachung von Demonstrationen in Österreich. Juridikum. Zeitschrift für Kritik, Recht, Gesellschaft, 28, 527-537.

Koranyi, J., \& Singelnstein, T. (2011). Rechtliche Grenzen für polizeiliche Bildaufnahmen von Versammlungen. Neue Juristische Wochenschrift, 64, 124-128.

Krasmann, S. (2005). Mobilität. Videoüberwachung als Chiffre einer Gouvernementalität der Gegenwart. In L. Hempel \& J. Metelmann (Hrsg.), Bild - Raum - Kontrolle. Videoüberwachung als Zeichen gesellschaftlichen Wandels (S. 308-324). Frankfurt a. M.: Suhrkamp.

Kutscha, M. (2011). Demonstrationen auf dem Bildschirm der Polizei. Kritische Justiz, 44, 223-232.

Lessenich, S. (2008). Die Neuerfindung des Sozialen. Der Sozialstaat im flexiblen Kapitalismus. Bielefeld: Transcript.

Link, J. (2005). „Normalisierungsgesellschaft“? „Kontrollgesellschaft“”? „Flexibler Normalismus“? Über einige aktuelle Gesellschaftskonzepte, mit einem Blick auf die „Reformen“. kultuRRevolution. Zeitschrift für angewandte Diskurstheorie, (49), 4-10.

Mann, S., Nolan, J., \& Wellman, B. (2003). Sousveillance. Inventing and using wearable computing devices for data collection in surveillance environments. Surveillance \& Society, 1, 331-355.

Marx, G. T. (2003). A tack in the shoe. Neutralizing and resisting the new surveillance. Journal of Social Issues, 59, 369-390.

Monahan, T. (2006). Counter-surveillance as political intervention? Social Semiotics, 16, 515-531.

Neumayer, C., \& Stald, G. (2014). The mobile phone in street protest. Texting, tweeting, tracking, and tracing. Mobile Media \& Communication, 2, 117-133.

Norris, C. (2005). Vom Persönlichen zum Digitalen. Videoüberwachung, das Panopticon und die technologische Verbindung von Verdacht und gesellschaftlicher Kontrolle. In L. Hempel \& J. Metelmann (Hrsg.), Bild - Raum - Kontrolle. Videoüberwachung als Zeichen gesellschaftlichen Wandels (S. 360-401). Frankfurt a. M.: Suhrkamp.

Norris, C., \& Armstrong, G. (1999). The maximum surveillance society. The rise of CCTV. Oxford: Berg. 
della Porta, D., \& Reiter, H. (1998). Introduction. The policing of protest in Western democracies. In D. della Porta \& H. Reiter (Hrsg.), Policing protest. The control of mass demonstrations in Western democracies (S. 1-32). Minneapolis: University of Minnesota Press.

della Porta, D., \& Tarrow, S. (2012). Interactive diffusion. The coevolution of police and protest behavior with an application to transnational contention. Comparative Political Studies, 45, 119-152.

della Porta, D., Reiter, H., \& Peterson, A. (2006). Policing transnational protest. An introduction. In D. della Porta, A. Peterson \& H. Reiter (Hrsg.), The policing of transnational protest (S. 1-12). Aldershot: Ashgate.

Reicher, S., Stott, C., Drury, J., Adang, O., Cronin, P., \& Livingstone, A. (2007). Knowledge-based public order policing. Principles and practice. Policing - A Journal of Policy and Practice, 1, 403-415.

Rikspolisstyrelsen (Hrsg.). (2013). Field study handbook. GODIAC - Good practice for dialogue and communication as strategic principles for policing political manifestations in Europe. Stockholm: Rikspolisstyrelsen.

Schreiber, M., \& Adang, O. (2008). Schwarze Blöcke und blau/grüne Maßnahmen - Über Gewalt bei Großereignissen und die Rolle der Polizei. Die Polizei, 12, 346-350.

Shaw, F. (2013). ,Walls of seeing“. Protest surveillance, embodied boundaries, and counter-surveillance at Occupy Sydney. Transformations. Journal of Media, Culture \& Technology, (23), Art. 4.

Short, E., \& Ditton, J. (1998). Seen and now heard. Talking to the targets of open street CCTV. British Journal of Criminology, 28, 404-428.

Singelnstein, T. (2014). Körperverletzung im Amt durch Polizisten und die Erledigungspraxis der Staatsanwaltschaften - aus empirischer und strafprozessualer Sicht. Neue Kriminalpolitik, 25, 15-27.

Starr, A., Fernandez, L. A., Amster, R., Wood, L. J., \& Caro, M. J. (2008). The impacts of state surveillance on political assembly and association: A socio-legal analysis. Qualitative Sociology, 31, 251-270.

Strauss, A., \& Corbin, J. (1998). Basics of qualitative research. Techniques and procedures for developing grounded theory. Thousand Oaks: Sage.

Ullrich, P. (2011). Gesundheitsdiskurse und Sozialkritik - Videoüberwachung von Demonstrationen. Zwei Studien zur gegenwärtigen Regierung von sozialen Bewegungen und Protest. München: Deutsches Jugendinstitut. http://www.dji.de/fileadmin/user_upload/bibs/9_14638_Videoueberwachung_ Ullrich_2012.pdf. Zugegriffen: Nov. 2016.

Ullrich, P. (2017). „Normalbürger“ versus „Krawalltouristen“. Polizeiliche Kategorisierungen von Demonstrationen zwischen Recht und soziologischem Ermessen. In K. Liebl (Hrsg.), Empirische Polizeiforschung XX. Polizei und Minderheiten (S. 61-97). Frankfurt a. M.: Verlag für Polizeiwissenschaft.

Ullrich, P. (2018). Videoüberwachung von Demonstrationen und die Definitionsmacht der Polizei. Zwischen Objektivitätsfiktion und selektiver Sanktionierung. Österreichische Zeitschrift für Soziologie, 43, 323-346.

Ullrich, P., \& Knopp, P. (2018). Protesters' reactions to video surveillance of demonstrations: Counter-moves, security cultures, and the spiral of surveillance and counter-surveillance. Surveillance \& Society, 16, 183-202.

Ullrich, P., \& Wollinger, G. R. (2011). A surveillance studies perspective on protest policing. The case of video surveillance of demonstrations in Germany. Interface. A Journal for and about Social Movements, 3, 12-38.

Wells, H., \& Wills, D. (2009). Individualism and identity. Resistance to speed cameras in the UK. Surveillance \& Society, 6, 259-274.

Welsh, B. C., \& Farrington, B. C. (2002). Crime prevention effects of closed circuit television. A systematic review. London: Home Office.

Wilson, D. J., \& Serisier, T. (2010). Video activism and the ambiguities of counter-surveillance. Surveillance \& Society, 8, 166-180.

Philipp Knopp geb. 1988. Dipl.-Soz., Universitätsassistent an der Universität Wien. Forschungsschwerpunkte: Politische Soziologie, Surveillance Studies, Protest- und Polizeiforschung. Ausgewählte Veröffentlichungen: (mit J. Ackermann, K. Behne, F. Buchta, M. Drobot) Metamorphosen des Extremismusbegriffes: Diskursanalytische Untersuchungen zur Dynamik einer funktionalen Unzulänglichkeit, 2015; Abstand halten: Zur Deutung gesellschaftlicher Krisen im Diskurs Pegidas, in T. Heim (Hrsg.), Pegida als Spiegel und Projektionsfläche, 2017. 
Peter Ullrich geb. 1976. Dr. phil., Dr. rer. med., Bereichsleiter „Soziale Bewegungen, Technik, Konflikte“ am Zentrum Technik und Gesellschaft, Technische Universität Berlin. Forschungsschwerpunkte: Politische Soziologie, kultur-, diskurs- und gouvernementalitätstheoretische Perspektiven auf soziale Bewegungen und Protest, Surveillance Studies und Polizeiforschung, wissenssoziologische Antisemitismusforschung. Ausgewählte Veröffentlichungen: Deutsche, Linke und der Nahostkonflikt. Politik im Antisemitismus- und Erinnerungsdiskurs, 2013; (mit B. Baumgarten, P. Daphi; Hrsg.) Conceptualizing culture in social movement research, 2014; (mit S. Alcántara, N. Bach, R. Kuhn) Demokratietheorie und Partizipationspraxis. Analyse und Anwendungspotentiale deliberativer Verfahren, 2016. 